ARTICLE

DOI: $10.1038 / s 41467-017-01266-0$

\title{
Coupling fission and exit of RAB6 vesicles at Golgi hotspots through kinesin-myosin interactions
}

Stéphanie Miserey-Lenkei , Hugo Bousquet ${ }^{1}$, Olena Pylypenko², Sabine Bardin", Ariane Dimitrov ${ }^{1}$, Gaëlle Bressanelli², Raja Bonifay², Vincent Fraisier ${ }^{3}$, Catherine Guillou' ${ }^{4}$, Cécile Bougeret ${ }^{5}$, Anne Houdusse (D) ${ }^{2}$, Arnaud Echard (iD ${ }^{6} \&$ Bruno Goud ${ }^{1}$

The actin and microtubule cytoskeletons play important roles in Golgi structure and function, but how they are connected remain poorly known. In this study, we investigated whether RAB6 GTPase, a Golgi-associated RAB involved in the regulation of several transport steps at the Golgi level, and two of its effectors, Myosin IIA and KIF20A participate in the coupling between actin and microtubule cytoskeleton. We have previously shown that RAB6-Myosin IIA interaction is critical for the fission of RAB6-positive transport carriers from Golgi/TGN membranes. Here we show that KIF2OA is also involved in the fission process and serves to anchor RAB6 on Golgi/TGN membranes near microtubule nucleating sites. We provide evidence that the fission events occur at a limited number of hotspots sites. Our results suggest that coupling between actin and microtubule cytoskeletons driven by Myosin II and KIF20A ensures the spatial coordination between RAB6-positive vesicles fission from Golgi/ TGN membranes and their exit along microtubules.

\footnotetext{
${ }^{1}$ Institut Curie, PSL Research University, CNRS, UMR 144, Molecular Mechanisms of Intracellular Transport, F-75005 Paris, France. ${ }^{2}$ Institut Curie, PSL Research University, CNRS, UMR 144, Structural Motility, F-75005 Paris, France. ${ }^{3}$ Institut Curie, PSL Research University, CNRS, UMR 144, Cell and Tissue Imaging Facility (PICT-IBiSA), F-75005 Paris, France. ${ }^{4}$ ICSN-CNRS, Gif Sur Yvette, F-91190, France. ${ }^{5}$ Biokinesis, Paris, F-75008, France. ${ }^{6}$ Institut Pasteur, CNRS UMR3691, Membrane Traffic and Cell Division, F-75015 Paris, France. Correspondence and requests for materials should be addressed to S.M.L. (email: stephanie.miserey-lenkei@curie.fr) or to B.G. (email: bruno.goud@curie.fr)
} 
T he microtubule (MT) and actin cytoskeletons play important roles in Golgi structure and function. It is now well established that intact MT network and the minus-end MT dynein motor are required for maintaining the Golgi structure. MT depolymerization causes Golgi ribbon fragmentation and Golgi membranes redistribution near endoplasmic reticulum (ER) exit sites. In addition, cis- and trans-Golgi membranes can nucleate and polymerize MTs. This allows the dynamic association of the Golgi complex with the MTOC (MT organizing center) and polarized secretion of Golgi-derived transport carriers toward the leading edge of migrating cells ${ }^{1}$. Actin depolymerization has less pronounced effect on Golgi structure, but leads to its compaction ${ }^{2}$. Several studies have illustrated that actin and actin-binding proteins, including motors of the myosin family, regulate early events of transport biogenesis at the Golgi complex such as protein sorting and membrane fission ${ }^{3-9}$.

An important challenge is therefore to better understand how the actin and MT cytoskeletal systems are connected at the Golgi level. Some players have been identified. Connections can be established via the formin family proteins, such as mDia-1, formin-like/FMNL 1 and 2, and INF2 that are present on Golgi membranes ${ }^{10-13}$. A WASP (Wiskott-Aldrich syndrome protein) homolog, WHAMM, directly interacts with MTs and was shown to play a role in the maintenance of
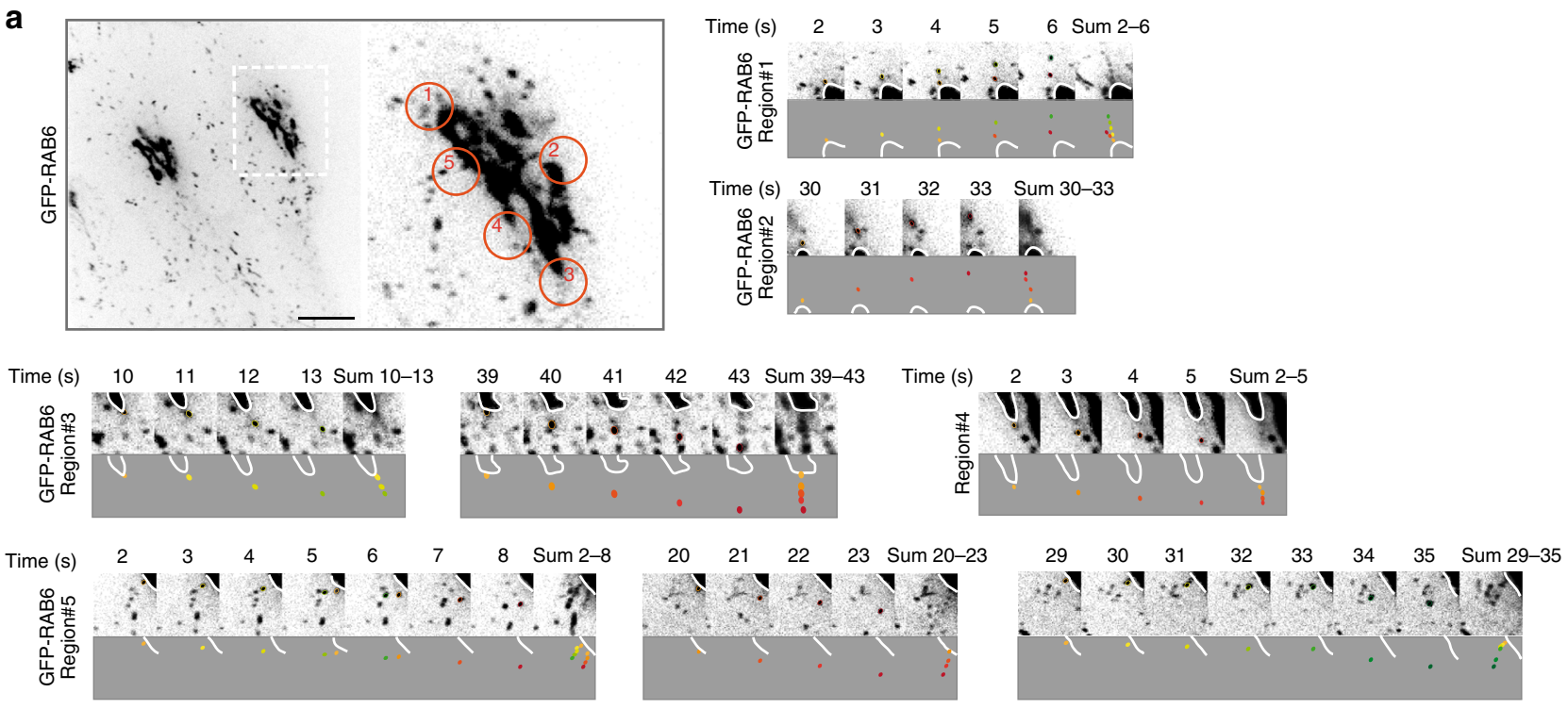

b

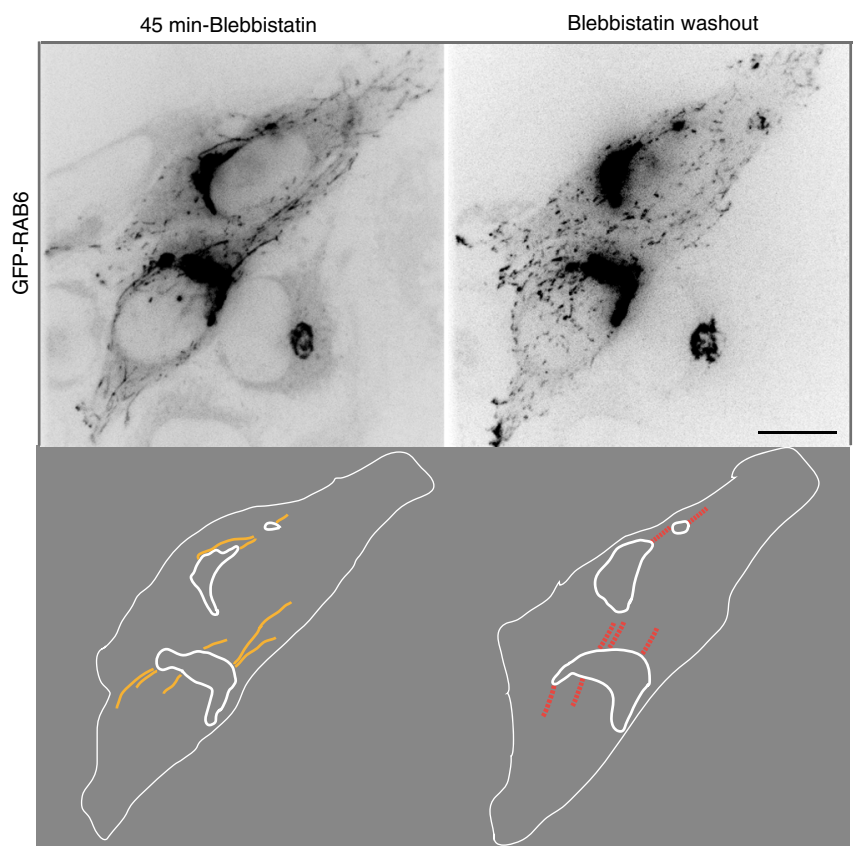

Fig. 1 GFP-RAB6-positive vesicles exit the Golgi at fission hotspots. a HeLa cells stably expressing GFP-RAB6 were imaged every second for 1 min (Supplementary Movie 1). Orange circles show the location of five fission hotspots. Details of each fission event for the five regions of hotspots are displayed. In each case, sequences of images are displayed at the top and a scheme of the events at the bottom. The Golgi region is highlighted by a white line. Vesicles exiting the Golgi are drawn and painted either yellow-green or orange-red. A sum of the events is shown at the end of each sequence. $\mathbf{b}$ Cells were treated for $45 \mathrm{~min}$ with para-nitro-blebbistatin. Then, para-nitro-blebbistatin was washed out. After recovery of normal GFP-RAB6 vesicles trafficking, the cells were imaged every second for $60 \mathrm{~s}$ (Supplementary Movie 3). A scheme of the images is displayed at the bottom. The Golgi regions are highlighted by a white line. Tubes are drawn in yellow. Regions where the GFP-RAB6-positive fission hotspots are found are drawn in red. Bars: $10 \mu \mathrm{m}$ 
Golgi structure as well as in ER to Golgi transport ${ }^{14}$. Other candidates are members of the Golgin family, such as the Drosophila golgin Lava lamp that interacts both with the dynein/ dynactin complex and spectrin $^{15}$, and the p230/golgin-245, shown to interact with MACF1, a giant protein that links MTs to the actin cytoskeleton ${ }^{16}$.

In this study, we investigated how RAB GTPases, key regulators of intracellular transport and membrane trafficking, and molecular motors control the coupling between actin and
MT cytoskeleton at the Golgi complex. One of the main functions of RAB GTPases is to recruit actin- or MT- based motors on transport carriers, allowing them to move along cytoskeletal tracks. This is the case for RAB6, the most abundant RAB at the Golgi that regulates several transport steps at the Golgi as well as Golgi homeostasis ${ }^{17-21}$. Two closely related RAB6 isoforms, RAB6A and RAB6A' are present on Golgi/TGN membranes ${ }^{6,22}$. In this manuscript, we will collectively call them RAB6. RAB6 was previously shown to directly or indirectly interact with several
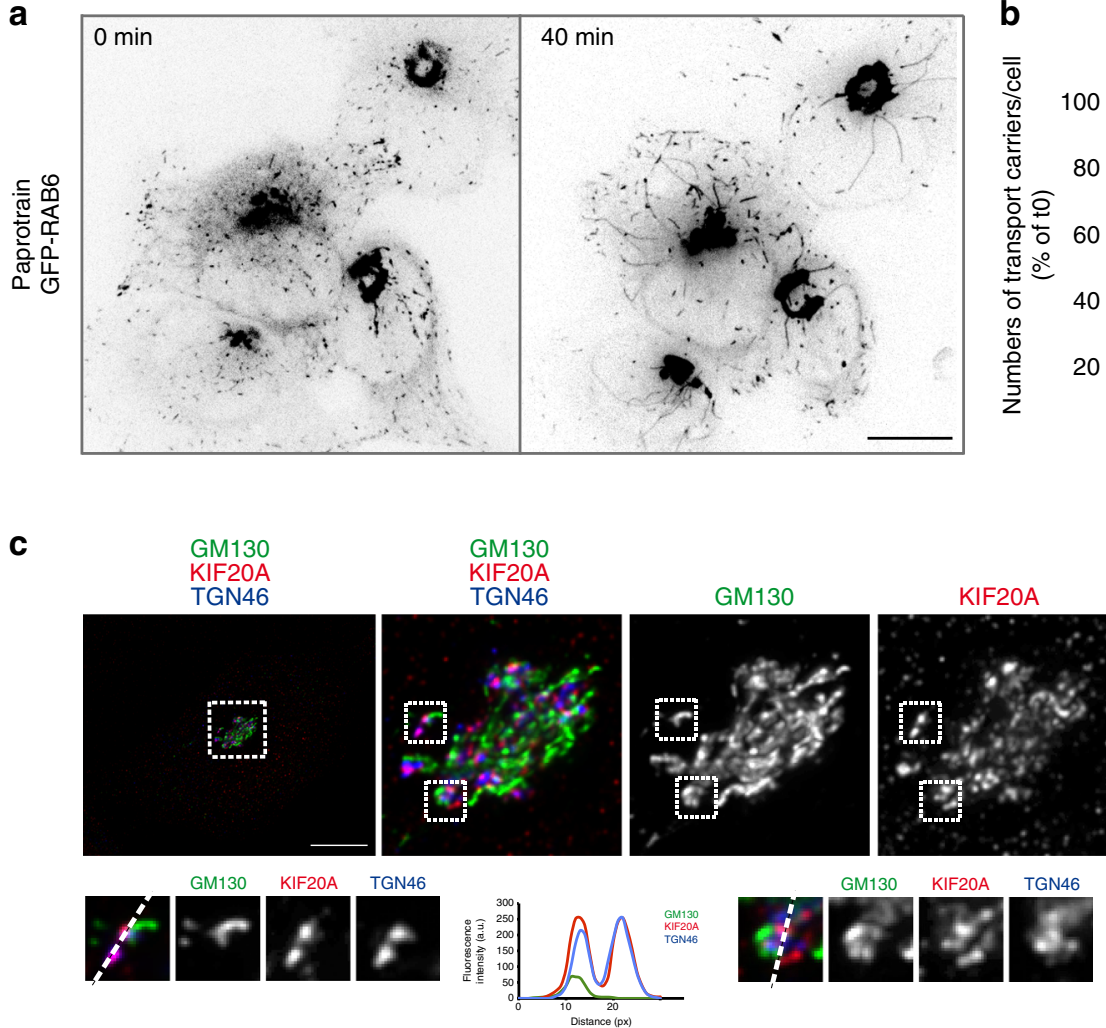

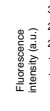
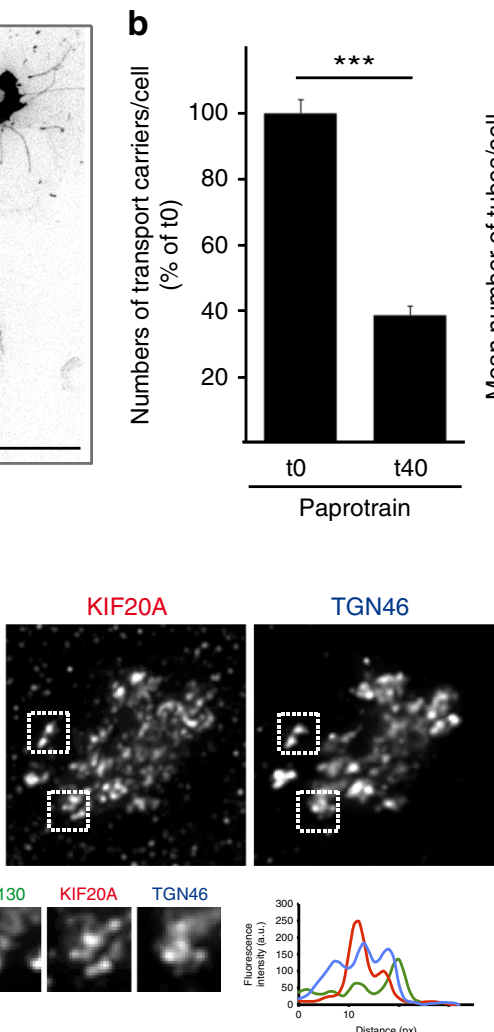

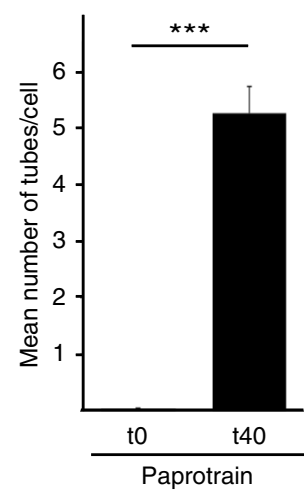

d GFP-RAB6
MLC-mCherry
KIF20A

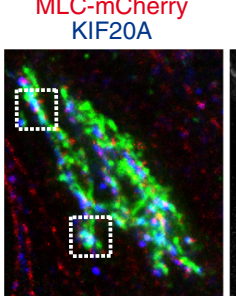
GFP-RAB6 MLC-mCherry
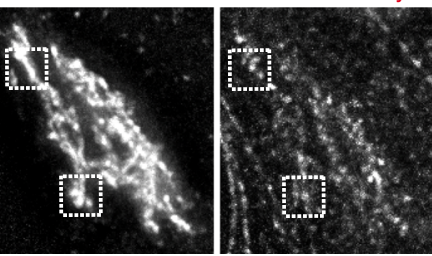

KIF20A
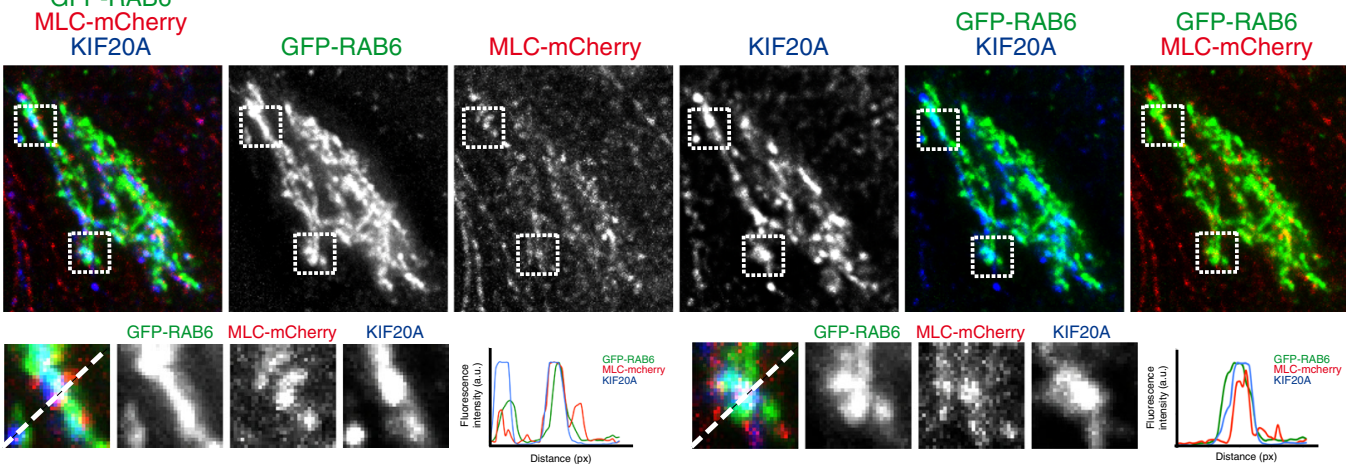

MLC-mCherry

KIF20A
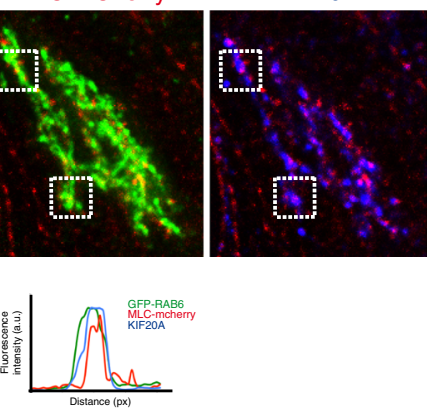

e

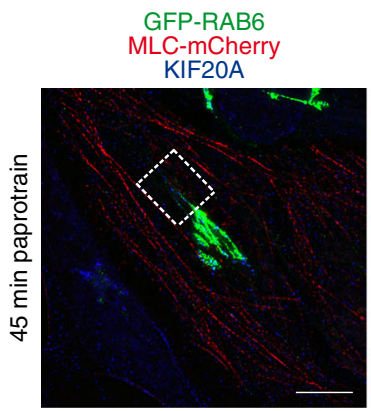

GFP-RAB6 KIF20A
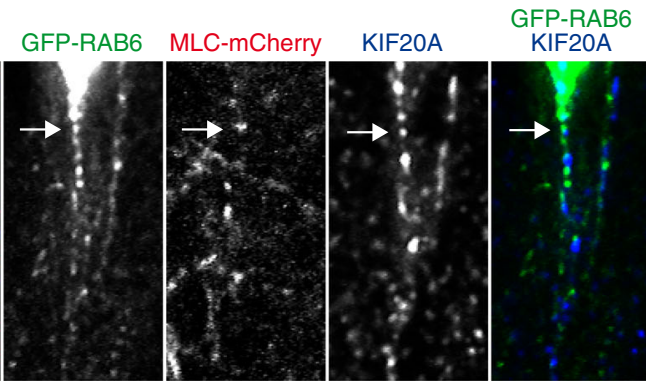

GFP-RAB6 MLC-mCherry

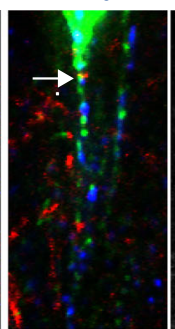


motors, including KIF5B, KIF20A (also known as Rabkinesin-6/ MKlp2), the dynein-dynactin complex (via Bicaudal-D), Myosin $\mathrm{II}$ and Myosin $\mathrm{Va}^{6,17,23-26}$. However, it remains unknown whether RAB6 acts as a platform to couple actin- and MTassociated motors in order to coordinate the function of MTs and actin in Golgi function.

The interaction between RAB6 and Myosin II is critical for the fission of RAB6-positive transport carriers from Golgi/TGN membranes ${ }^{6}$. Here, we show that KIF20A is also involved in the fission process. The coupling between actin and MT cytoskeleton driven by Myosin II and KIF20A ensures the spatial coordination of RAB6-positive vesicles formation at fission hotspots sites and their exit from Golgi/TGN membranes along MTs.

\section{Results}

RAB6-positive vesicles exit the Golgi complex at fission hotspots. We have previously shown that RAB6 and Myosin II are implicated in the fission of RAB6-positive transport carriers at the Golgi complex ${ }^{6}$. The inhibition of this process leads to the formation of long membrane tubes connected to the Golgi complex ${ }^{6}$. Detailed analysis of time-lapse microscopy of HeLa cells stably expressing GFP-RAB6 (Supplementary Fig. 1, Supplementary Movie 1) now revealed that RAB6-positive vesicles exit the Golgi complex in defined areas (Fig. 1a, Supplementary Fig. 1 and Supplementary Movie 1). We named them Golgi fission hotspots. A detailed illustration of Golgi fission hotspots for one Golgi is displayed in Fig. 1a. At the optical microscopy resolution, the Golgi fission hotspots are seen at the extremities rather than at the flatter regions of the Golgi. Over 60-s movies, we observed the existence of $6.4 \pm 0.4$ fission hotspots per Golgi $(n=13$ Golgi) and measured a total of $12.5 \pm 1.3$ vesicles per min exiting the Golgi complex at fission hotspots ( $n=13$ Golgi). This indicates that 1-2 RAB6-positive vesicles exit the Golgi complex at fission hotspots per min (Fig. 1a). The formation of a vesicle usually takes $1 \mathrm{~s}$ (Fig. 1a; example in region \#1, between time 3 and 4; example in region \#5, between time 4 and 5). Movies performed on the same Golgi complex at 10-min interval revealed that the fission hotspots are stable over time (Supplementary Fig. 1, Supplementary Movie 2). If RAB6 vesicles exit the Golgi at preferential sites, one expects that these sites are similar to the ones where the membrane tubes are formed after inhibition of the fission process. To address this point, GFP-RAB6 cells were treated with the Myosin II inhibitor blebbistatin to inhibit fission and to allow the appearance of membrane tubes. Then, blebbistatin was washed out and the Golgi fission hotspots were localized using detailed analysis of time-lapse movies over a 60-s movie. The location of fission hotspots was similar to that of the RAB6positive membrane tubes obtained after inhibition of the fission process (Fig. 1b, Supplementary Movie 3). Altogether, these results highlight the existence of RAB6-positive Golgi fission hotspots on the Golgi complex.
KIF20A is involved in the fission process of RAB6-positive vesicles. To decipher the molecular mechanisms of the fission process of RAB6-positive vesicles from Golgi membranes, we looked for proteins interacting with both Myosin II and RAB6. KIF20A, that we previously identified as a RAB6 effector present on Golgi membranes ${ }^{25}$, appeared as an interesting candidate since it was recently shown to directly interact with Myosin $\mathrm{II}^{27,28}$.

We originally proposed that KIF20A was responsible for the movement of RAB6-positive vesicles along MTs, since dominant negative mutants of KIF20A reduced trafficking ${ }^{25}$. Alternatively, KIF20A might be involved in the generation of RAB6 vesicles. Good evidence later showed that RAB6 vesicles move thanks to KIF $5 \mathrm{~B}^{6,17}$. Consistent with these results, we found that KIF20A inhibition using paprotrain, a specific chemical inhibitor of KIF20A motor activity ${ }^{29}$, did not affect the speed of RAB6positive vesicles $(0.91 \pm 0.04 \mu \mathrm{m} / \mathrm{s}$ in control, $0.89 \pm 0.04 \mu \mathrm{m} / \mathrm{s}$ in paprotrain-treated cells, $n=47$ ). To address the possible function of KIF20A in the generation of RAB6 vesicles, we performed time-lapse video-microscopy experiments in HeLa cells stably expressing GFP-RAB6 following KIF20A inhibition with paprotrain or its depletion by short interfering RNA (siRNA) (Fig. 2a,b, Supplementary Fig. 2). Interestingly, in both cases, we observed the appearance of long tubules connected to the Golgi complex (Fig. 2a, b, Supplementary Fig. 2, Supplementary Movie 4) and a strong reduction in the number of RAB6-positive vesicles in the cytoplasm (Fig. 2b, Supplementary Fig. 2, Supplementary Movie 4). This phenotype, strikingly similar to the one observed after Myosin II inhibition or its depletion ${ }^{6}$, suggests that KIF20A is involved in the fission process of RAB6-positive vesicles from Golgi membranes. Fissions defects were also observed using a second inhibitor BKS0349 which is derived from paprotrain and displays higher affinity (Supplementary Fig. 2) or by overexpressing KIF20 constructs lacking the motor domain (Supplementary Fig. 3). To further confirm that the motor activity of KIF20A is required for the fission of RAB6-positive vesicles, we overexpressed at moderate levels (to avoid bundling of MTs) in control or KIF20A siRNA-treated cells, either wild-type or the K165A mutant of KIF20A (Supplementary Fig. 4). This conserved lysine in the P-loops of kinesins and myosins is absolutely required for nucleotide binding and motor activity ${ }^{30-32}$. As expected, expression of GFP-KIF20A WT rescued the fission defects resulting from KIF20A depletion (Supplementary Fig. 4). In contrast, GFP-KIF20A-K165A expressing cells still displayed membrane tubules connected with the Golgi and a decreased number of vesicles in the cytoplasm (Supplementary Fig. 4). Interestingly, GFP-KIF20A-K165A by itself induced the appearance of Golgi-connected membrane tubules and fission defects, indicating that this construct acts as a dominant negative mutant (Supplementary Fig. 4). Altogether, these results demonstrate that the ATPase activity of KIF20A is required for the fission process of Rab6-positive vesicles.

Fig. 2 Inhibition of KIF20A function inhibits the fission of Rab6 transport carriers from the Golgi. GFP-RAB6, Myosin II, and KIF20A are co-localized on dotted structures that correspond to the sites of fission. a HeLa cells stably expressing GFP-RAB6 were imaged by time-lapse microscopy, and images of the same cell before (t0) or $40 \mathrm{~min}(\mathrm{t} 40)$ after paprotrain $(25 \mu \mathrm{M})$ treatment are presented (Supplementary Movie 4). Paprotrain was used as an inhibitor of KIF20A function. $\mathbf{b}$ Quantification of the number of transport carriers and of Golgi-connected tubules in cells treated as indicated in (a) (mean \pm SEM, $n$ $=38$ cells). ${ }^{\star \star \star} P<10^{-10}$ (Student's $t$ test). c Staining of endogenous GM130 (green), KIF20A (red), and TGN46 (blue) in HeLa cells indicates a higher colocalization of KIF20A with the trans-Golgi marker TGN46 as compared to the cis-Golgi marker GM130 (see higher magnifications (boxes) on the bottom). Line profiles of the GM130 (green), the KIF2OA (red), and the TGN46 (blue) fluorescence intensities (arbitrary units) along the white-dashed arrow. Bar, $10 \mu \mathrm{m}$. d Staining of GFP-RAB6 (green), MLC-mCherry (red), and endogenous KIF20A (blue) in HeLa cells indicates a partial co-localization of the three proteins on dotted structures at the Golgi complex (see higher magnifications (boxes) on the bottom). Line profiles of the GFP-RAB6 (green), the MLC-mCherry (red), and the KIF20A (blue) fluorescence intensities (arbitrary units) along the white-dashed arrow. Bar, $10 \mu \mathrm{m}$. e Staining of GFP-RAB6 (green), MLC-mCherry (red), and endogenous KIF20A (blue) in HeLa cells treated for 45 min with paprotrain indicates a co-localization (arrow) of the three proteins at the base of a GFP-RAB6-positive tube. Bar, $10 \mu \mathrm{m}$ 


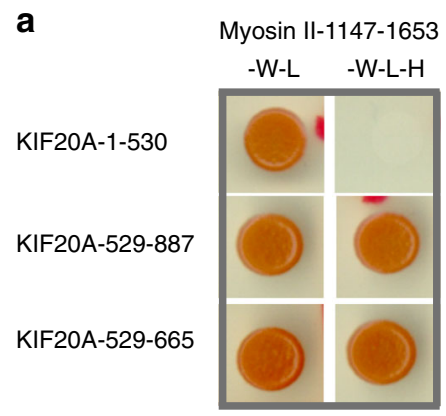

C

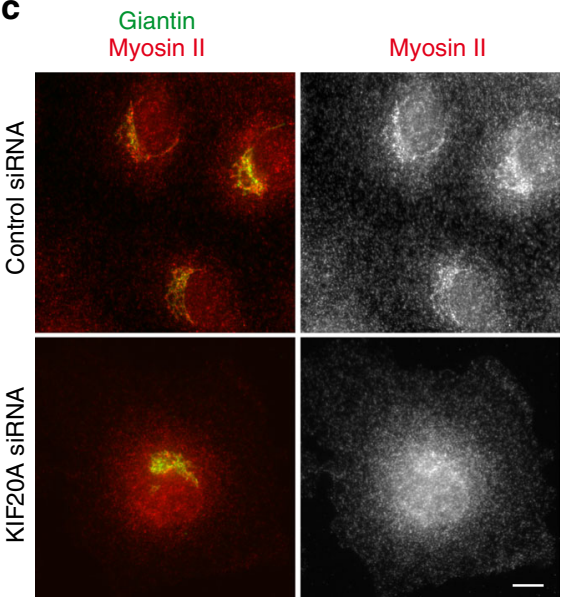

b
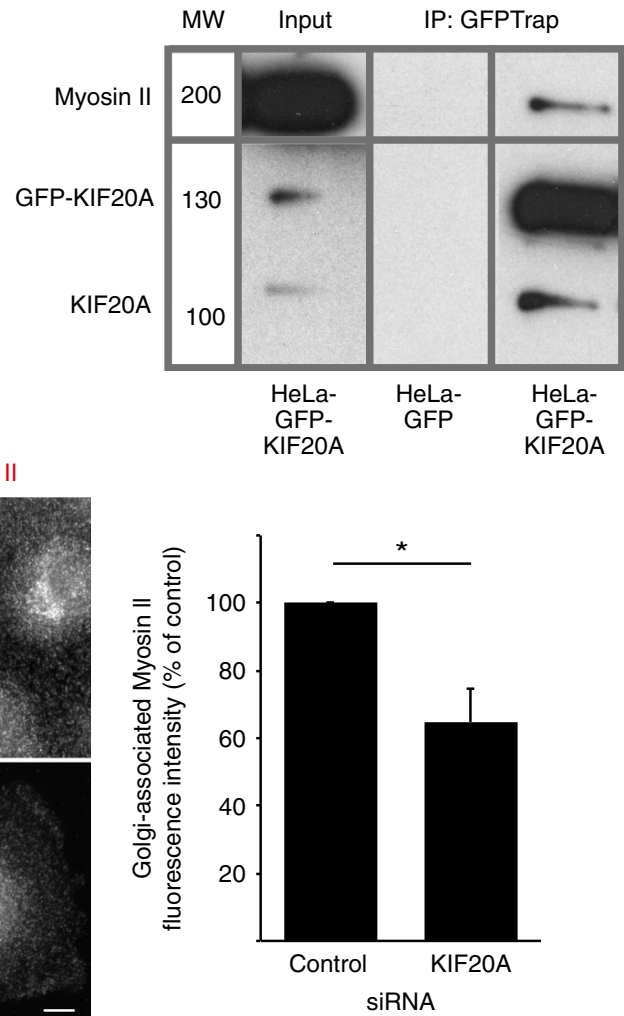

Fig. 3 KIF20A interacts with Myosin II and stabilizes Myosin II at the Golgi. a Yeast two-hybrid interactions between the tail domain of Myosin II (1147-1653 fragment) and different domains of KIF20A. The Saccharomyces cerevisiae reporter strain L40 was co-transformed with a plasmid encoding fusion proteins to detect interactions between amino acids 1147 and 1653 of human myosin IIA heavy chain and the motor domain (1-530), the tail domain (529-887), and the RAB6 binding domain (RBD) (529-665) of KIF20A. Growth on medium lacking histidine (-W -L -H) indicates an interaction between the encoded proteins. b Endogenous Myosin II is pulled-down by GFP-KIF20A. GFP-KIF20A or GFP-transfected HeLa cell extracts were immunoprecipitated using the GFP-trap system. Myosin II bound to GFP-KIF2OA was revealed by western blot analysis using anti-Myosin II antibody. GFPKIF20A and KIF20A were revealed by anti-KIF20A antibody. Note that endogenous KIF20A is found in the IP fraction because it likely can form dimers with GFP-KIF2OA. Input represents a 5\% load of the total cell extracts used in all conditions. c Staining for endogenous Giantin (green) and endogenous Myosin II using the AD7 antibody (red) in Rat Clone 9 cells 3 days after transfection with specific KIF20A siRNAs. Bar, 10 um. Of note, KIF20A is required for cytokinesis and KIF20A depletion leads to binucleated cells ${ }^{56,57}$. Quantification of Golgi-associated Myosin II fluorescence intensity in cells treated as described above (mean $\pm \mathrm{SEM}, n=23-66$ cells). ${ }^{\star} P=0.02$ (Student's $t$ test). MW molecular weight in $\mathrm{kDa}$

Finally, as already reported after RAB6 or Myosin II depletion/ inhibition $^{6}$, the trafficking of the secretory cargo VSV-G from the Golgi to the plasma membrane was impaired after KIF20A inhibition (Supplementary Fig. 2).

KIF20A, RAB6, and Myosin II are co-localized at Golgi fission hotspots. KIF20A can be visualized on fixed cells associated with the Golgi complex ${ }^{25}$ (Fig. 2c). The co-localization was more pronounced with the TGN marker TGN46 than with the cis-Golgi marker GM130 (Fig. 2c). We validated the Golgi localization of KIF20A using two different antibodies (Supplementary Fig. 5) as well as in a GFP-KIF20A cell line (Supplementary Fig. 5) and we showed that the Golgi signal decreased following KIF20A depletion by siRNA (Supplementary Fig. 5).

We performed a detailed analysis of the extent of co-localization between RAB6, Myosin II, and KIF20A on these Golgi fission hotspots. GFP-RAB6, Myosin II, and KIF20A were found to colocalize on dotted structures (Fig. 2d). On average, $6.3 \pm 0.7$ of these dots were found per Golgi $(n=21$ Golgi). Following KIF20A inhibition with paprotrain, dots containing the three proteins were found at the base of membrane tubes, suggesting that these dots correspond to the sites of fission (Fig. 2e). To further confirm this hypothesis, we treated the cells with blebbistatin to induce the appareance of membrane tubules and then washed out the drug.
As previously shown ${ }^{6}$, this leads to membrane fission along the tubules close to membrane bulges that are labeled by RAB6, cargos and F-actin. We observed an accumulation of KIF20A close to the membrane bulges, indicating the presence of KIF20A at the fission sites (Supplementary Fig. 5).

KIF20A displays two Myosin II binding sites. KIF20A was recently shown to directly interact with Myosin $\mathrm{II}^{27,28}$. The Myosin II binding site has been restricted to the last 60 amino acids of KIF20A and has been shown by co-immunoprecipitation experiments to be the main contributor in Myosin II binding ${ }^{28}$. Using yeast two-hybrid experiments, we confirmed this interaction and identified a second binding site corresponding to the KIF20A-529-665 fragment (Fig. 3a and Supplementary Fig. 6). This fragment was previously characterized as the RAB6 binding domain of KIF20A (RBD) ${ }^{25}$. The tail domain of Myosin II thus interacts with the tail domain of KIF20A via two binding sites (Fig. 3a and Supplementary Fig. 6). However, these two binding sites likely act independently since overexpression of the KIF20A529-887 domain does not lead to higher fission defects than the overexpression of the KIF20A-529-665 or KIF20A-796-887 domains alone (Supplementary Fig. 3). Consistent with the yeast two-hybrid experiments, GFP-KIF20A and endogenous Myosin II could be immunoprecipitated in the same complexes (Fig. 3b). 
KIF20A recruits and/or stabilizes Myosin II at the Golgi complex. We then investigated whether KIF20A could be involved in the recruitment and/or stabilization of Myosin II at the Golgi complex. In order to quantify the amount of Myosin II on the Golgi, we used a rat cell line and a Myosin II-specific antibody, named AD7, whose specificity was previously validated $^{6,7,33}$. KIF20A depletion reduced by $35 \%$ the amount of Myosin II associated with the Golgi complex (Fig. 3c). Similar results were obtained using a Myosin light chain specific antibody (Supplementary Fig. 7). Altogether, the above results show that a

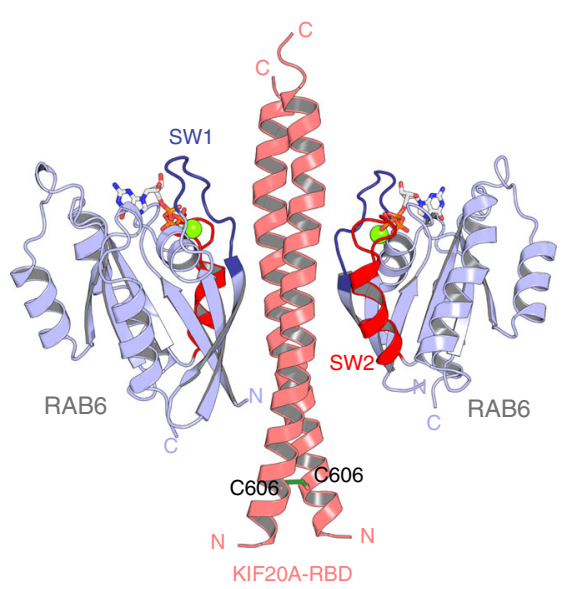

b

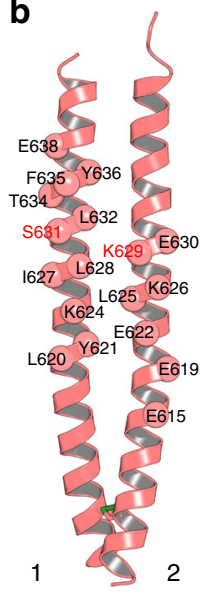

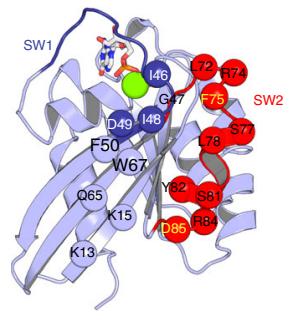

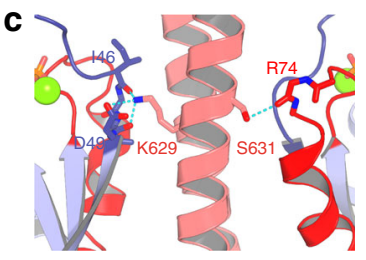

d

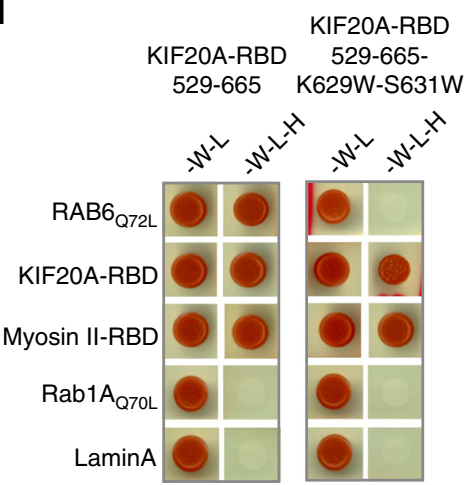

f Giantin KIF20A

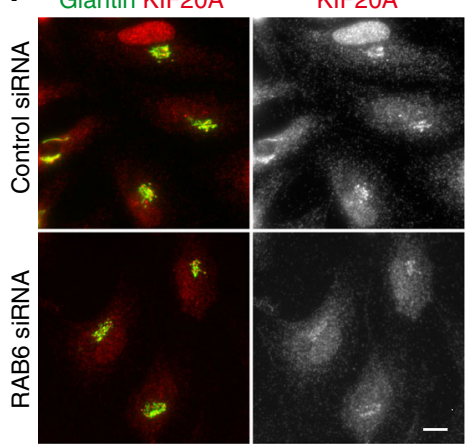

e
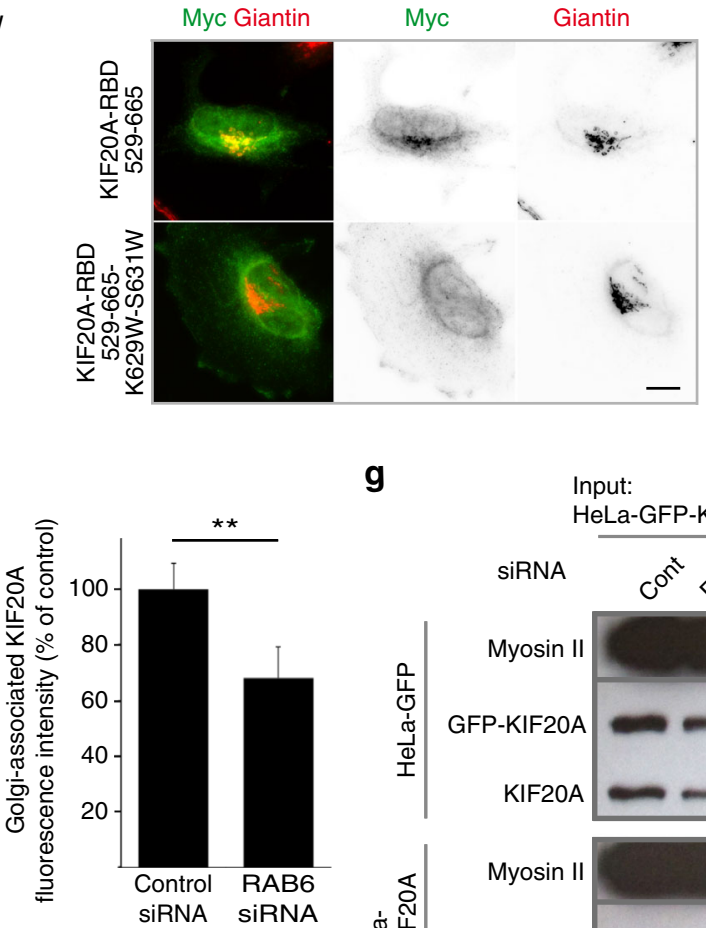

g

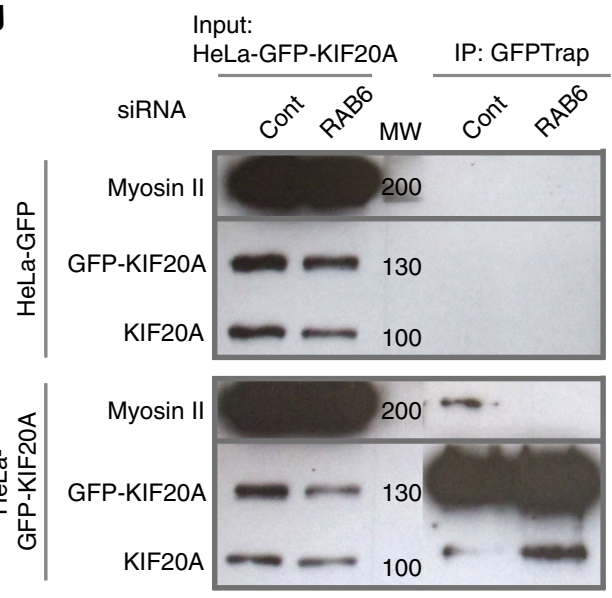

h
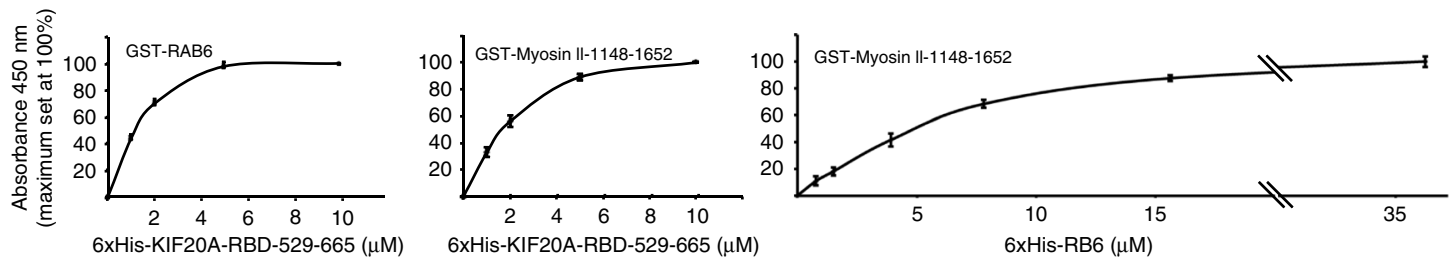
both KIF20A and Myosin II act together in the fission of RAB6positive vesicles from the Golgi complex.

RAB6 is required for the Golgi association of KIF20A. KIF20A interacts directly with RAB6 $6^{25}$. To further characterize this interaction, we solved the crystal structure of the complex between RAB6 and KIF20A (Fig. 4a-c). The RAB6:KIF20A structure reveals that the 603-645 residues of KIF20A represent the RBD. KIF20A-RBD is a dimer composed of two parallel alpha helices that form a right-handed coiled-coil additionally stabilized by an inter-helical cysteine bridge (Fig. 4a). Both RBD helices participate in RAB6 binding, two RAB6 molecules binding at opposite sides of the dimer (Fig. 4a-c). The RAB6:KIF20A interface is formed by complementary surfaces of the partners (Fig. 4b) that mediate hydrophobic and polar contacts between the molecules. KIF20A K629 and S630 residues are located in the central part of the RAB6-binding site (Fig. 4b, c) and form polar contacts with the partner (Fig. 4c). Prediction based on the structure indicates that substitution of these polar residues by bulky hydrophobic residues should substantially alter the RAB6-binding site. Indeed, the KIF20A (529-665) fragment with K629W and S630W mutations lost its capacity to interact with RAB6 Q72L by yeast two-hybrid (Fig. 4d). We have previously shown that overexpressed KIF20A-RBD can be recruited to the Golgi complex ${ }^{25}$. A myc-tagged version of the KIF20A-529-665K629W-S630W fragment overexpressed in HeLa cells remained mostly cytosolic in contrast to the Golgi-localized wild-type form (Fig. 4e), demonstrating the importance of the RAB6:KIF20A direct interaction for KIF20A recruitment to Golgi. The mutations do not affect KIF20A-RBD dimerization and importantly its ability to interact with the tail domain of Myosin II (Fig. 4d). This suggests that RAB6 and Myosin II may bind to the KIF20A dimeric coiled-coil (603-665) in different ways. Since this site has two RAB6-binding sites, one of them might be used for Myosin II binding rather than for RAB6 binding and the tri-partite complexes between KIF20A, RAB6, and Myosin II can still be formed. Finally, depletion of RAB6 reduced by $40 \%$, the amount of KIF20A associated with the Golgi complex (Fig. 4f), indicating that RAB6 contributes to the recruitment and/or the stabilization of KIF20A at the Golgi. Since the recruitment of KIF20A on the Golgi only partially depends on RAB6, it is likely that additional mechanisms are implicated or alternatively that the Golgi-associated pool of KIF20A is very stable.

To further understand this process, we tested whether RAB6 is required for KIF20A/Myosin II interaction. Co-immunoprecipitation experiments showed that the complex between KIF20A and Myosin II was lost in RAB6-depleted cells (Fig. 4g). Similar results were obtained in MEF cells derived from RAB6 conditional KO mice embryos and incubated in vitro with tamoxifen to deplete RAB6 (Supplementary Fig. 7).

We next measured the relative binding affinities of RAB6 for Myosin II, RAB6 for KIF20A, and KIF20A for Myosin II using a previously described solid-phase assay ${ }^{34}$. These experiments revealed that RAB6 and Myosin II-1148-1652 bind to KIF20ARBD-529-665 with similar affinities in the micromolar range (Fig. 4h). On the other hand, RAB6 binds to Myosin II-1148-1652 with around five times lower affinity (Fig. 4h). The fact that RAB6 and Myosin II display the highest affinities for KIF20A suggests that KIF20A is central for the interaction between RAB6 and Myosin II. A likely scenario is that RAB6 first recruits KIF20A, which then binds to Myosin II (see model on Fig. $6 c$ and discussion).

KIF20A limits RAB6 diffusion at the Golgi ensuring RAB6 localization at fission hotspots. At the metaphase/anaphase transition, KIF20A was shown to stabilize the central spindle thanks to its additional C-terminal MT-binding domain and to promote the localization on MTs of several proteins, including Plk1 and Aurora $\mathrm{B}^{35-37}$. A tempting hypothesis is that KIF20A fulfils a similar role on Golgi membranes to anchor RAB6 near MTs, which would favor the loading of RAB6 vesicles onto MTs.

To test this hypothesis, we performed FRAP (fluorescence recovery after photobleaching) experiments in HeLa cells stably expressing GFP-RAB6 (Fig. 5a, b, Supplementary Movie 5). After FRAP on Golgi extremities, a wave of RAB6 fluorescence coming from the core of the bleach area to the extremity of the Golgi was observed (Fig. 5b, Supplementary Movie 5). Following KIF20A inhibition with paprotrain, the wave of diffusion corresponding to RAB6 fluorescence was faster (Fig. 5c, Supplementary Movie 5) as exemplified at time $5 \mathrm{~s}$. The half-time recovery after photobleaching was also reduced by $30 \%$ as compared to control cells (Fig. 5d, e), reflecting a higher mobility of GFP-RAB6 on Golgi membranes (Fig. 5d, e).

The above results indicate that KIF20A limits the diffusion of RAB6 molecules on Golgi membranes and confine them into membrane areas close to MTs.

KIF20A co-localizes with Golgi-associated growing MTs on Golgi membranes. After fission from Golgi membranes, RAB6positive vesicles are transported along MTs to the cell periphery ${ }^{17}$. Golgi membranes can nucleate and polymerize MTs that serve in particular to sustain polarized secretion ${ }^{38-40}$. We thus

Fig. 4 Definition of the RAB6-BD of KIF20A. RAB6 partially recruits KIF20A on the Golgi complex. RAB6 is required for KIF20A-Myosin II interaction. Relative binding affinities between RAB6, KIF20A, and Myosin II. a-c KIF20A-RAB6-binding domain (KIF20A-RBD) is a dimer composed of parallel helices that form a right-handed coiled-coil stabilized by an inter-helical cysteine bridge and two RAB6 molecules bind on opposite sides of the dimer $\mathbf{b}$. The RAB6: KIF20A interface makes hydrophobic and polar contacts between the molecules b, $\mathbf{c}$, in particular K629 and S631, are buried in the interface and involved in direct interactions at the center of the interface. d Yeast two-hybrid interactions between the KIF20A-RBD-529-665 fragment or KIF20A-RBD-529-665K626W-S631W fragment with RAB6, Myosin II-RBD, and KIF20A-RBD. The Saccharomyces cerevisiae reporter strain L40 was co-transformed with a plasmid encoding fusion proteins to detect interactions between the KIF20A-RBD-529-665 fragment or KIF20A-RBD-529-665-K626W-S631W fragment and RAB6-Q72L, KIF20A-RBD, Myosin II-RBD, RAB1Q70L, and Lamin A. Growth on medium lacking histidine (-W -L -H) indicates an interaction between the encoded proteins. e Golgi association of the KIF20A-RBD-529-665 fragment or KIF20A-RBD-529-665-K626W-S631W fragments. Staining for endogenous Giantin (red) and overexpressed myc-tagged KIF20A-RBD-529-665 fragment or KIF20A-RBD-529-665-K626W-S631W fragment. f Staining of endogenous Giantin (green) and KIF2OA (red) in HeLa cells 3 days after transfection with specific RAB6 siRNAs. Quantification of Golgi-associated KIF20A fluorescence intensity in cells treated as described above (mean $\pm \mathrm{SEM}, n=34-37$ cells). ${ }^{\star \star} P<10^{-3}$ (Student's $t$ test). Bar, $10 \mu \mathrm{m}$. $\mathbf{g}$ RAB6 is required for KIF20A/Myosin II interaction. GFP-KIF20A or GFP expressing HeLa cell extracts treated for 3 days with control or RAB6-specific siRNAs were immunoprecipitated using the GFP-trap system. Myosin II bound to GFP-KIF20A was revealed by western blot analysis using anti-Myosin II antibody. Input represents a $5 \%$ load of the total cell extracts used in all conditions. $\mathbf{h}$ 96-well plates were coated with recombinant GST-RAB6 or GST-Myosin II-11481652 and incubated with increasing amounts of recombinant $6 \times$ His-KIF20A-RBD-529-665 or $6 \times$ His-RAB6, as indicated (solid-phase assay). After washes, KIF20A-RBD-529-665 or RAB6 proteins bound to RAB6 or Myosin II-1148-1652 were detected (arbitrary units) using anti- $6 \times$ His antibodies and a chromogenic substrate (mean $\pm \mathrm{SEM}, n=3$ experiments). No binding to GST-GFP alone was detected. MW molecular weight in kDa 
a

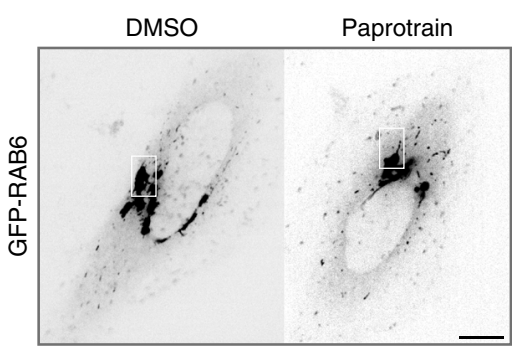

b

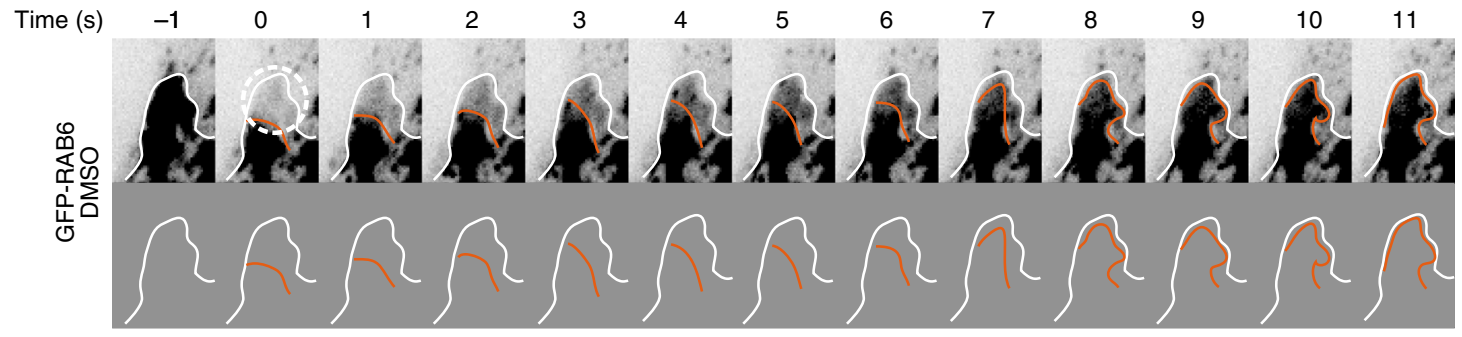

C

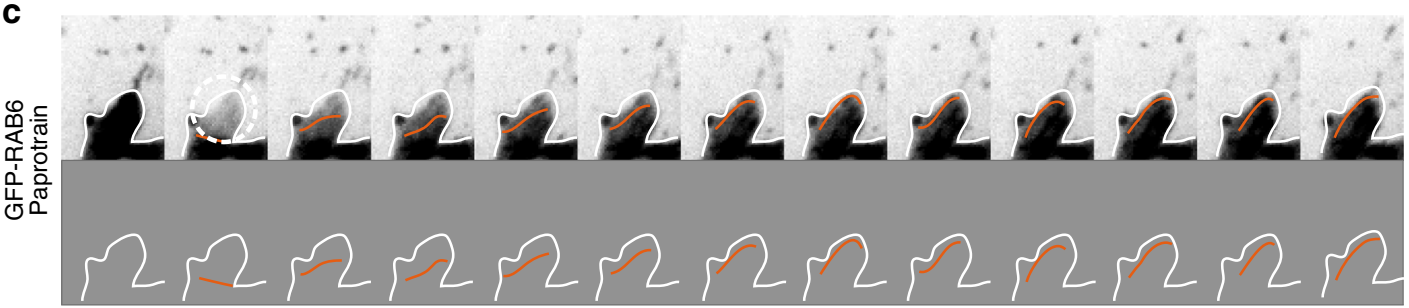

d

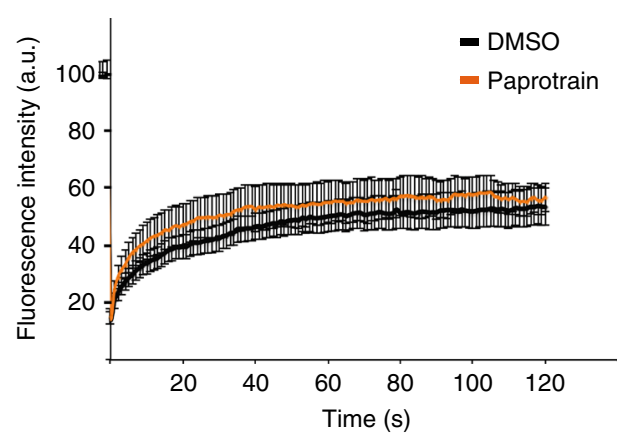

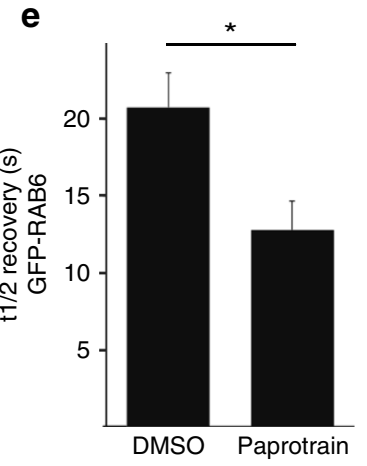

Fig. 5 GFP-RAB6 diffuses on Golgi membranes and KIF2OA inhibition affects the diffusion of GFP-RAB6. a Images of GFP-RAB6 expressing cells treated with DMSO or with paprotrain for 30 min. HeLa cells expressing GFP-RAB6 were bleached in a 30 pixels circular region of the Golgi apparatus (white circle) and then imaged for 2 min by spinning disk microscopy. Sequence of images of GFP-RAB6 in Golgi of a control cell (b) and a paprotrain-treated cell (c) before and after photobleaching. The round region delimited by a dotted line was photobleached immediately after the first image $(-1)$. In both cases, images are displayed at the top and schematics of the images at the bottom. The orange line represents the recovery of the GFP-RAB6 fluorescence. In $\mathbf{b}$, the photobleached area rapidly recovered fluorescence. The fluorescence flows from the center of the Golgi to the extremity of the bleached area. In $\mathbf{c}$, the photobleached area recovered fluorescence twice faster than in $\mathbf{b}$ (compare t5). $\mathbf{d}$ The fluorescence recovery after photobleaching was measured in three independent experiments and plotted for GFP-RAB6 treated with DMSO (black) or with paprotrain (orange) ( $n=17-19$ Golgi). e Half-life recovery of GFP-RAB6 fluorescence in control cells and cells treated with paprotrain (mean $\pm \mathrm{SEM}, n=17-19$ cells). ${ }^{*} P=0.01$ (Student's $t$ test)

wondered if KIF20A interacts with MTs that polymerize on Golgi membranes. First, we observed that after nocodazole treatment and washout, tubulin dots corresponding to reforming MTs co-localize with KIF20A (Fig. 6a). Second, Myosin II and RAB6 are found co-localized on peripheral dots with GCC185, a TGN Golgin known to recruit the MT + TIP CLASP proteins to the TGN, allowing the nucleation and formation of Golgi $\mathrm{MTs}^{38}$ (Fig. 6b). Quantification indicated that there are $7.51 \pm 0.46$ dots of triple co-localization per Golgi ( $n=25$ Golgi). Altogether, these results indicate that RAB6, KIF20A, and Myosin II are co-localized on dots corresponding to places of polymerization of MTs originating from Golgi membranes.

\section{Discussion}

The main finding of this study is the existence of preferential sites for the fission of RAB6-positive post-Golgi secretory vesicles on Golgi/TGN membranes. Hotspots for the fission of endocytic vesicles and ER-derived vesicles (ER-exit sites) as well as exocytic hotspots ${ }^{41}$ have been previously described at the plasma or ER 
membranes, but to our knowledge never on Golgi/TGN membranes.

We measured around six hotspots per Golgi. Remarkably, this number corresponds to the number of spots where RAB6, Myosin II, and KIF20A co-localize and to the number of membrane tubes observed after the inhibition of Myosin II $(n=5$, ref. $\left.{ }^{6}\right)$, that of KIF20A ( $n=5$, Fig. 2$)$ or RAB6 depletion $(n=4$, ref. ${ }^{6}$ ). This suggests that a connection between actin and MT cytoskeletons established by an actin-based motor and a MTbased motor that interact with each other is necessary for the a
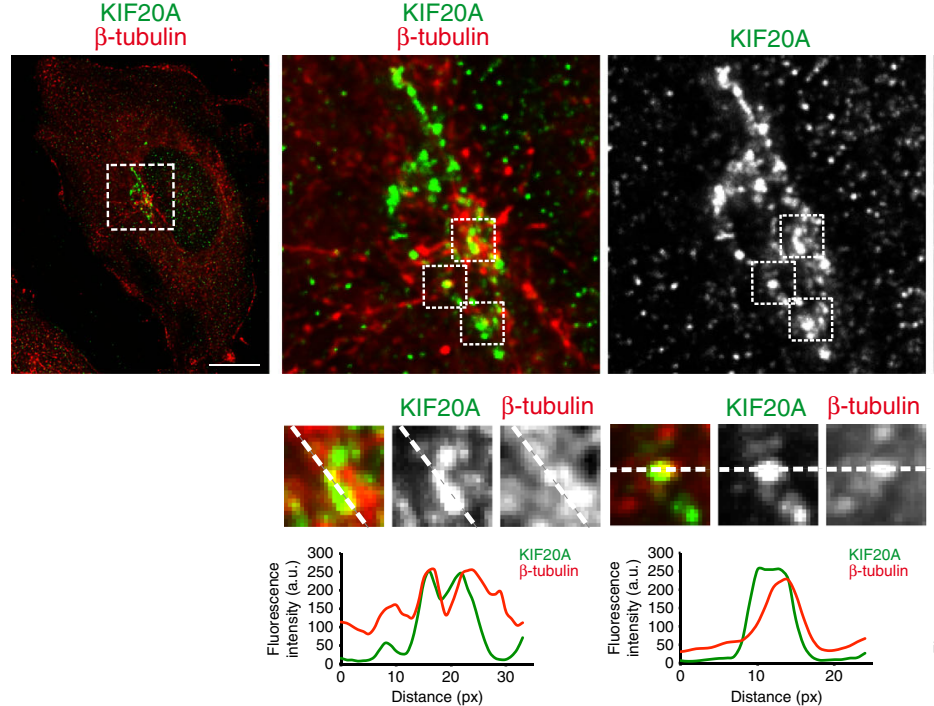

KIF20A $\beta$-tubulin
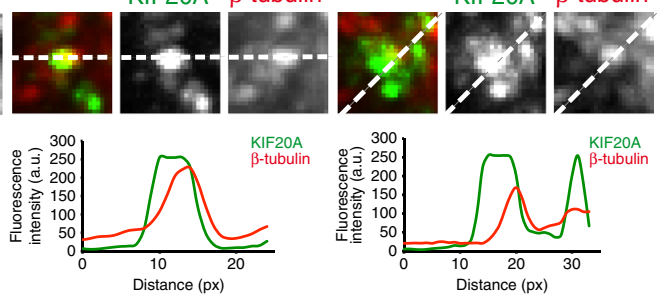
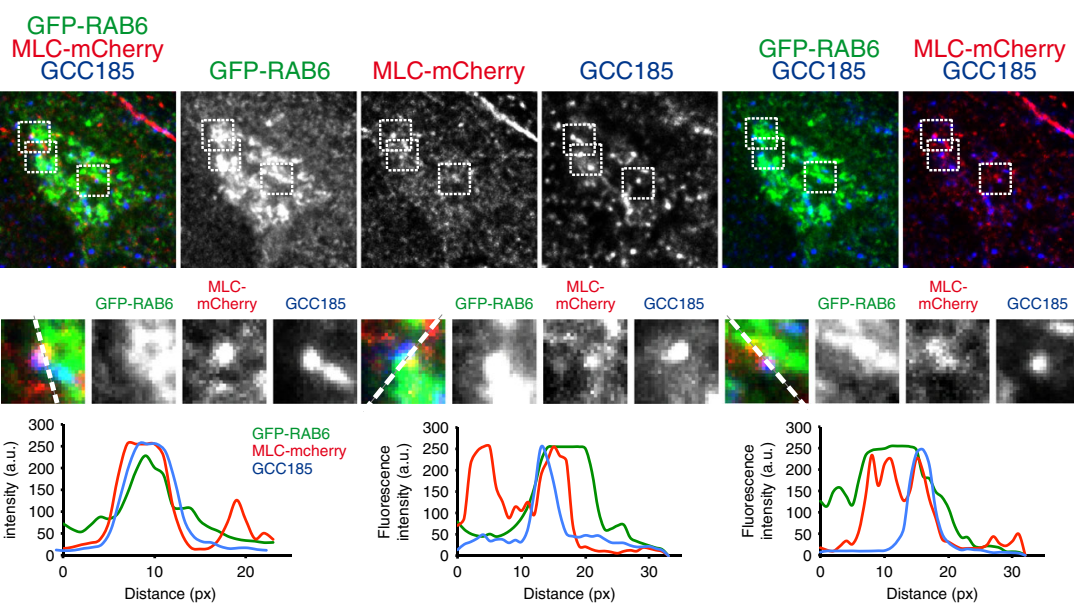

C

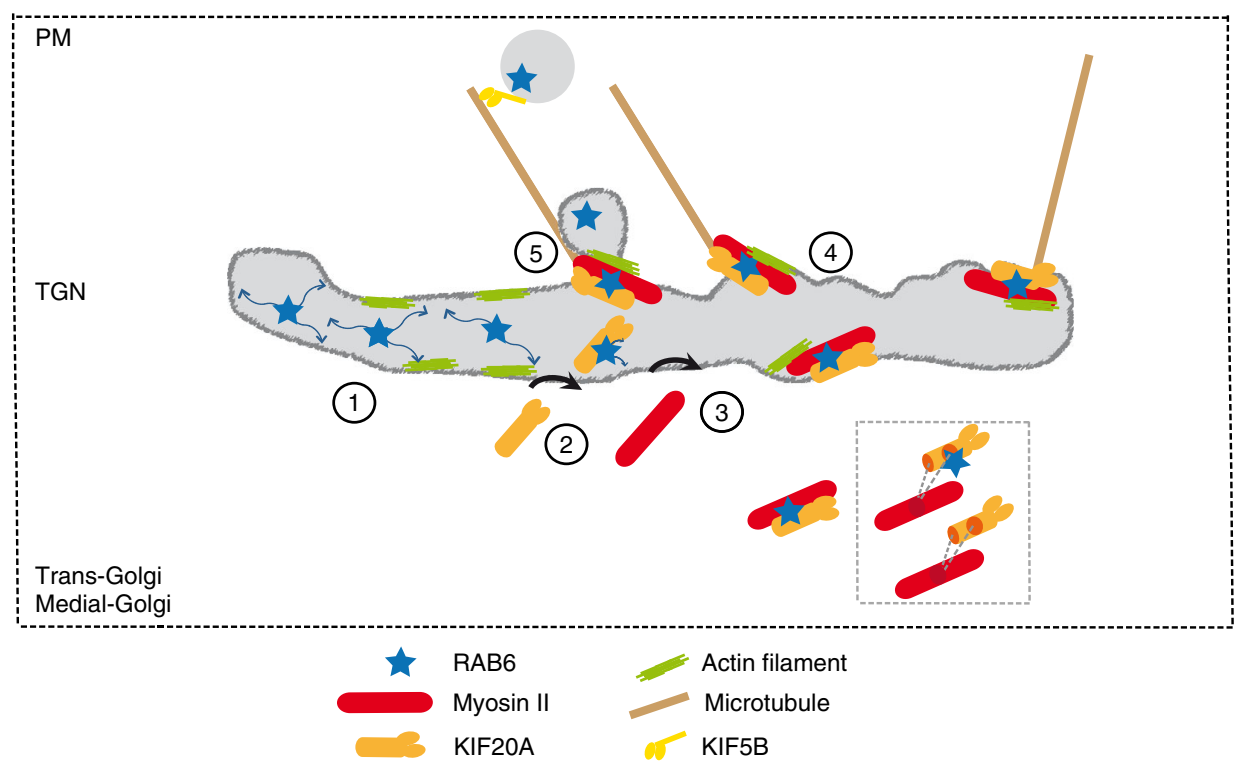


generation of Golgi fission hotspots. It is likely however that additional mechanisms determine where and when hotspots can form. A tentative hypothesis is that some RAB6 molecules are concentrated in domains, maybe enriched in specific lipids such as PtdIns-4-phosphate. The existence of such domains has been postulated $^{42}$ but not yet demonstrated. In addition, other fission promoting molecular complexes such as CtBP1-S/BARS, members of the 14-3-3 family, phosphoinositide kinase PI4KIII $\beta$, and lysophosphatidic acid acyltransferase $\delta$ have been identified ${ }^{43-45}$. It will be important to test in future experiments whether KIF20A/RAB6/Myosin II complexes are physically or functionally related to these complexes. Another important issue will be to investigate the relationships between the budding and fission machineries of RAB6-positive vesicles. RAB6 depletion leads to the accumulation of budding profiles on Golgi membranes ${ }^{46}$ that are coated with clathrin and $\mathrm{COPI}^{18}$. This suggests that the fission machinery driven by Myosin II and KIF20A is distinct from that required for budding.

The sequence of events that can be envisioned for the generation of fission hotspots is the following (Fig. 6c). RAB6 depletion leads to a decrease of Golgi-associated KIF20A, indicating that RAB6 participates, at least in part, to KIF20A recruitment on Golgi/TGN membranes. This would allow the clustering of RAB6 molecules and surrounding membranes to MTs. In support of this hypothesis, we showed that KIF20A inhibition increases the diffusion rate of RAB6 on Golgi/TGN membranes. Such a role for KIF20A is reminiscent of the one proposed during mitosis where KIF20A promotes the MT-dependent localization of Plk1, Aurora B, and Cdc14A to the central spindle ${ }^{35}$. Myosin II would then be recruited by KIF20A and RAB6 and would drive the fission of RAB6-positive transport carriers from Golgi/TGN membranes. An alternative scenario is that Myosin II is first recruited and then interacts with KIF20A. This is however unlikely since depletion of KIF20A decreases the amount of Myosin II associated with Golgi/TGN membranes.

RAB6-positive transport vesicles move along MTs toward the cell periphery and the plasma membrane to convey secretory cargoes such as VSV-G or NPY ${ }^{6,17}$. Whether the MTs used originate from MTOC or Golgi membranes is unknown, but our observations suggest that at least part of these MTs could originate from Golgi membranes. Indeed, KIF20A co-localizes with MTs that regrow from Golgi membranes. In addition, GCC185, a golgin involved in the formation of Golgi-associated MTs, could also be visualized on structures containing RAB6 and Myosin II. Importantly, the motor activity of KIF20A appears to be required for a normal fission process (Supplementary Figs. 2-4). As KIF20A is not the kinesin moving RAB6-positive vesicles along MTs (KIF5B is responsible for this, see refs. ${ }^{6,17}$ ), two possibilities can be envisioned: the motor activity of KIF20A could be important to generate a level of membrane tension required for vesicle fission or alternatively it could be essential for the local organization of MTs to favor the right positioning of the fission machinery.

We previously showed that RAB6 controls the fission of its own transport carriers, which provides a mechanism for coordinating several events involved in the biogenesis of transport vesicles. Our study illustrates that in addition RAB6 could select a subset of MTs for transport of its own transport carriers. It will be interesting to see whether this is a unique mechanism or if other RAB GTPases that interact with actin and MT-based motors could function in the same way for the biogenesis of transport vesicles.

\section{Methods}

Antibodies and reagents. The following reagents were used: ParaNitroblebbistatin (OPTOPHARMA Ltd), Y-27632 (Calbiochem), ML7 (Calbiochem), Paprotrain (Enzo Life Sciences). The following antibodies were used for biochemical experiments: Mouse anti-GM130 (BD Biosciences, \#610822), sheep anti-TGN46 (AbD Serotec, AHP500), rabbit anti-KIF20A (ref. ${ }^{25}$; Bethyl Laboratory, \#A300-879A; proteintech, \#15911-1-AP), goat anti-KIF20A (Santa Cruz Biotechnology, sc-104954), rabbit anti-RAB6 (ref. ${ }^{47}$ and Santa Cruz Biotechnology, sc-310), human anti-Giantin (Recombinant antibody platform, Institut Curie, Paris, France); rabbit anti-myc (Santa Cruz Biotechnology, sc-789), rabbit anti-MHC (Myosin II Heavy Chain) (Bethyl Laboratory, A304-490A), human anti $\beta$-tubulin (Recombinant antibody platform, Institut Curie), rabbit antiGCC185 (P. Gleeson, University of Melbourne, Australia), mouse anti-actin (Sigma, A-2228), rabbit ant-Cul3 (Bethyl Laboratory, A-301-110A), mouse antipoly-histidine antibody (Recombinant antibody platform, Institut Curie). The plasmids encoding the following fusion proteins were used: GFP-RAB6 (F. Perez Institut Curie), mCherry-RAB6, MLC-mCherry (A.M. Lennon-Dumenil, Institut Curie), GFP-KIF20A-529-665 ${ }^{25}$, GFP-KIF20A-529-887 ${ }^{25}$, GFP-KIF20A-25-887 (I.M. Yu, Institut Curie).

Cell culture and transfection. HeLa ${ }^{25}$ and Rat Clone 9 cells (ATCC \#CRL1439) were grown in DMEM medium (Gibco BRL) supplemented with $10 \%$ fetal bovine serum, $100 \mathrm{U} / \mathrm{ml}$ penicillin/streptomycin, and $2 \mathrm{mM}$ glutamine. Both cell lines were tested free for mycoplasma contamination. Cells were seeded onto 6-well plates, $18-$, or $12-\mathrm{mm}$ glass coverslips or Fluorodish and grown for $24 \mathrm{~h}$ before transfection. HeLa cells stably expressing GFP-RAB6A' or GFP-KIF20A (KIF20A-BAC, a kind gift from A. Hyman, Dresden, Germany) were cultivated in the presence of $50 \mu \mathrm{g} / \mathrm{ml}$ geneticin (Gibco BRL). For expression of the constructs used in this study, HeLa cells were transfected using either the calcium phosphate precipitation method or X-tremeGENE9 (Roche) following the manufacturer's instructions. For silencing experiments, HeLa and Rat Clone 9 cells were transfected with the corresponding siRNA once (in the case of RAB6A/A' siRNAs) or twice at $24 \mathrm{~h}$ interval (in the case of KIF20A siRNAs) using HiPerFect (Qiagen) following the manufacturer's instructions. For rescue experiments, cells were transfected with GFP-KIF20A or GFP-KIF20A-K165A $24 \mathrm{~h}$ after transfection with KIF20A siRNAs Forty-eight hours after this second round of transfection, cells were proceeded for video-microscopy experiments. The N-terminal 25 amino acids truncated version of KIF20A was used because of better expression efficiency.

RNA interference. The sequences of the siRNAs used in this study are the following: human RAB6A/A': (GACAUCUUUGAUCACCAGA) ${ }^{48}$. These oligonucleotides were obtained from Sigma (Paris, France). For silencing human KIF20A, different sequences were used: one described in ref. ${ }^{36}$ (obtained from Sigma) and specific SMART pools chemically synthesized by Dharmacon Research,

Fig. 6 KIF2OA co-localizes with growing microtubules on Golgi membranes. a HeLa cells were incubated for 45 min at $4^{\circ} \mathrm{C}$ to promote microtubule depolymerization (without affecting the Golgi morphology) and then incubated for $3 \mathrm{~min}$ at RT to allow microtubule repolymerization. Staining of endogenous KIF2OA (green) and $\beta$-tubulin (red) in HeLa cells indicates a partial co-localization of KIF20A with growing microtubules from Golgi membranes (see higher magnifications (boxes) on the bottom). Line profiles of the KIF20A (green) and the $\beta$-tubulin (red) fluorescence intensities (arbitrary units) along the white dashed line. Bar, $10 \mu \mathrm{m}$. b Staining of GFP-RAB6 (green), MLC-mCherry (red), and endogenous GCC185 (blue) in HeLa cells indicates a partial co-localization of the three proteins on dotted structures at the Golgi complex (see higher magnifications (boxes) on the bottom). Line profiles of the GFP-RAB6 (green), the MLC-mCherry (red), and the GCC185 (blue) fluorescence intensities (arbitrary units) along the white dashed line. Bar, $10 \mu \mathrm{m}$. c Schematic of the sequence of events that can be envisioned for the generation of fission hotspots at the TGN. (1) RAB6 diffuses on Golgi/TGN membranes. (2) RAB6 participates in the recruitment and stabilization of KIF20A. When bound to KIF20A, RAB6 diffusion is decreased allowing the localization and anchoring of RAB6 molecules to sites of growing Golgi-associated microtubules. (3) Myosin II is recruited by KIF20A and RAB6. Either a complex between RAB6 and KIF20A is required for Myosin II recruitment, or KIF20A acts alone. Myosin II can be recruited by the KIF20ARBD-529-665 domain in complex or not with RAB6 or through the 796-887 domain. (4) KIF20A, RAB6, and Myosin II, in association with actin filaments and microtubules, define a Golgi fission hotspot. There are around six hotspots per Golgi. (5) Myosin II and actin then drive the fission of RAB6-positive transport carriers from Golgi/TGN membranes. RAB6-positive vesicles are then transported along microtubules to the plasma membrane thanks to KIF5B 
Inc. SiRNA targeting luciferase (CGUACGCGGAAUACUUCGA) was used as a control and was obtained from Sigma.

Yeast two-hybrid experiments. A yeast two-hybrid screen of a human placental cDNA library with mouse KIF20A-529-887 as a bait was performed by Hybrigenics SA (www.hybrigenics.com). Several clones corresponding to human non-muscle Myosin II heavy chain gene have been isolated. These clones correspond to nucleotides 3984-4413 of the CDS of human Myosin IIA. Different sequences corresponding to nucleotides 3744-4122, 3744-3984, 3987-4413 of the CDS of human Myosin IIA have been amplified by PCR and inserted into the SmaI site of yeast two-hybrid pGADGH vector. Yeast two-hybrid experiments were performed as described in ref. ${ }^{6}$ except that the LexA fusion proteins correspond to different domains of KIF20A described in ref. ${ }^{49}$.

Construction of KIF20A mutants. For KIF20A-529-665-K629W-S631W mutants: single-point mutations were inserted in the mouse KIF20A sequence at position 1884, 1887, and 1893 using the QuickChange mutagenesis kit (Agilent). For mCherry-KIF20A-796-887: the 2388-2661 fragment of mouse KIF20A was amplified by PCR and inserted into a mCherry vector allowing the mammalian and bacterial expression of mCherry-KIF20A-796-887. For GFP-KIF20A-25-665 the 75-1995 fragment of human KIF20A was amplified by PCR and inserted into a pOPIN-GFP vector (Addgene) allowing the mammalian expression of GFPKIF20A-25-665. For GFP-KIF20A-25-887-K165A: single-point mutation was inserted in the mouse KIF20A sequence at position 495 using the QuickChange mutagenesis kit (Agilent).

Co-immunoprecipitation and western blot experiments. To test the interaction between GFP-KIF20A and endogenous Myosin II, HeLa cells transfected or not with RAB6 siRNA were trypsinized, washed once in PBS, and incubated on ice for $60 \mathrm{~min}$ in a lysis buffer: $25 \mathrm{mM}$ Tris $\mathrm{pH} 7.5,50-100$ or $200 \mathrm{mM} \mathrm{NaCl}$, and $0.1 \%$ NP40. Cells were then centrifuged $10 \mathrm{~min}$ at $10,000 \times g$ to remove cell debris. Extracts were then processed for co-immunoprecipitation using GFP-trap (Chromotek) following manufacturer's instructions.

To test the interaction between RAB6 and endogenous Myosin II and KIF20A, MEF cells treated or not with Tamoxifen for $96 \mathrm{~h}$ (for details see ref. ${ }^{46}$ ) were trypsinized, washed once in PBS, and incubated on ice for $60 \mathrm{~min}$ in a lysis buffer: $25 \mathrm{mM}$ Tris $\mathrm{pH} 7.5,50-100$ or $200 \mathrm{mM} \mathrm{NaCl}, 0.1 \% \mathrm{NP} 40$, protease inhibitor cocktail (Sigma). Cells were then centrifuged $10 \mathrm{~min}$ at $10,000 \times \mathrm{g}$ to remove cell debris. Extracts were then processed for co-immunoprecipitation using $2 \mu \mathrm{g}$ of anti-KIF20A antibody coupled to Protein G-Sepharose beads for $4 \mathrm{~h}$ at $4{ }^{\circ} \mathrm{C}$ in lysis buffer. A Rabbit anti-Culin3 antibody was used as control IgG. Beads were washed four times in lysis and then processed for western-blotting.

For western-blotting experiments, cells were processed as in ref. ${ }^{6}$. Cell solubilization was performed in $25 \mathrm{mM}$ Tris $\mathrm{pH} 7.5,50 \mathrm{mM} \mathrm{NaCl}, 0.1 \% \mathrm{NP} 40$, and a protease inhibitor cocktail (Sigma). The following primary antibodies were used: rabbit anti-MHC (Covance; 1:2000), rabbit anti-RAB6 (Santa Cruz 1:1000 or ref. ${ }^{47}$ 1:2000), mouse anti-GFP (Roche; 1:1000), rabbit anti-KIF20A (Bethyl or A174 ${ }^{25}$; 1:1000). Secondary Horseradish Peroxidase (HRP)-coupled antibodies were from Jackson Laboratories.

Immunofluorescence microscopy. HeLa or Rat Clone 9 cells grown on coverslips were fixed either in $4 \%$ paraformaldehyde (PFA) for $15 \mathrm{~min}$ at RT, in methanol $\left(2 \mathrm{~min},-20^{\circ} \mathrm{C}\right)$ or in TCA $\left(10 \%\right.$ TCA $20 \mathrm{~min}$ at $4{ }^{\circ} \mathrm{C}$, followed by a $3 \mathrm{~min}$ permeabilization in PBS, $0.1 \%$ Triton X-100). For mAD7 staining, cells were treated for $2 \mathrm{~min}$ with $0.1 \%$ Triton X-100 after fixation with PFA. Cells were then processed for immunofluorescence as previously described ${ }^{48}$. The following primary antibodies were used: human anti-Giantin (1:200, ref. $\left.{ }^{50}\right)$, mouse anti-GM130 (1:1000), mouse AD7 anti-myosin II (1:50 to $1: 200$, ref. $\left.{ }^{33}\right)$, rabbit anti-GCC185 (1:100), rabbit anti-KIF20A (1:2000 to $1: 8000$, ref. $\left.{ }^{25}\right)$, goat antiKIF20A (1:2000 to $1: 8000)$, rabbit anti-MLC (1:200, Cell Signaling), human and rabbit anti- $\beta$ tubulin (1:200, ref. $\left.{ }^{50}\right)$, and sheep anti-TGN46 (1:1000). Fluorescently coupled secondary antibodies were obtained from Jackson. Coverslips were mounted in Mowiol and examined under a three-dimensional deconvolution microscope (Leica DM-RXA2), equipped with a piezo z-drive (Physik Instrument) and a $100 \times 1.4$ NA-PL-APO objective lens for optical sectioning or a DMRA Leica microscope with a $63 \times$ objective lens. Three-dimensional or one-dimensional multicolor image stacks were acquired using the Metamorph software (MDS) through a cooled CCD camera (Photometrics Coolsnap HQ). For deconvolution, cell images were acquired as described in ref. ${ }^{51}$.

Time-lapse fluorescence microscopy. Transfected cells were grown either on glass bottom Fluorodish or on glass coverslips and transferred, just before observation, to custom-built aluminium microscope slide chambers (Ludin chamber, LIS). Time-lapse imaging was performed at $37^{\circ} \mathrm{C}$ using a spinning-disk microscope mounted on an inverted motorized microscope (Nikon TE2000-U) through a $100 \times 1.4$ NA PL-APO objective lens. The apparatus is composed of a Yokogawa CSU-22 spinning-disk head, a Roper Scientific laser launch, a Photometrics Coolsnap HQ2 CCD camera for image acquisition and Metamorph software (MDS) to control the setup. Acquisition parameters were $100 \mathrm{~ms}$ exposure for GFP channel and $100 \mathrm{~ms}$ for mCherry channel. Laser was set to $30 \%$ in each case. Images shown in figures correspond to the maximal intensity projection through the $Z$ axis performed with the Image J software (NIH Image). For FRAP analyses, HeLa cells stably expressing GFP-RAB6 were maintained in culture medium in glass bottom Fluorodish cell culture dishes and imaged on a similar spinning disk microscope equipped with FRAP head (Errol and Roper). Images were collected before bleaching of a rounded 30 pixels region of the Golgi apparatus every second for $5 \mathrm{~s}$ and after photobleaching every $1 \mathrm{~s}$ for 2-3 min. Images were processed using the Metamorph software. After correction of the photo-bleaching due to acquisition, the background was subtracted. The intensity of fluorescence was then normalized and plotted on the graph.

\section{Quantification of the number of transport carriers and membrane tubes.}

A total of 16-50 transfected cells were imaged for each condition. When cells were treated with drugs, quantifications were performed on the same cells, before and after drug treatment. The maximal intensity projection through the $z$ axis of each image stack was done using the Image J software. The number of transport carriers (corresponding to transport carriers moving at least on three following snapshot) and tubes (connected to the Golgi complex) was manually counted using the cell counter macro from ImageJ (NIH Image).

\section{Quantification of Myosin II and KIF20A Golgi-associated fluorescence} intensity. Images of control or depleted cells were acquired using the same parameters, without automatic scaling and gain adjustment, avoiding saturated pixels. In all cases, the Golgi area was defined by the Giantin labeling, and the absolute intensity of Golgi-associated Myosin II or KIF20A in this area was measured using Image J software (NIH Image). In the case of KIF20A depleted cells, since cells are multinucleated and have a bigger Golgi, the KIF20A Golgiassociated fluorescence intensity was normalized to the size of the Golgi.

Recombinant protein purification and solid-phase assays. GST fused to Myosin II-1148-1652 was expressed in the BL21 (DE3) strain of Escherichia coli after induction with $500 \mu \mathrm{M}$ isopropyl- $\beta$-d-thiogalactopyranoside at $20^{\circ} \mathrm{C}$ overnight. Cells were lysed in PBS, 2 mM $\beta$-mercaptoethanol, protease inhibitor and lysosyme by cell disruption. The GST fusion proteins were affinity-purified using Glutha-

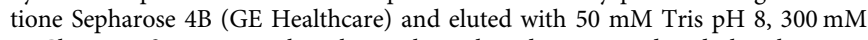
$\mathrm{NaCl}, 2 \mathrm{mM} \beta$-mercaptoethanol, $5 \%$ glycerol, and $10 \mathrm{mM}$ reduced glutathione. Purified proteins were then dialyzed against a solution containing $20 \mathrm{mM}$ Hepes $\mathrm{pH} 7.5,100 \mathrm{mM} \mathrm{NaCl}, 5 \mathrm{mM} \mathrm{MgCl}$. GST fused to wild-type RAB6A was expressed in the BL21 codon plus (DE3)-RIL strain of E. coli after induction with $400 \mu \mathrm{M}$ isopropyl- $\beta$-d-thiogalactopyranoside at $20^{\circ} \mathrm{C}$ overnight. Cells were lysed by cell disruption in $50 \mathrm{mM}$ Hepes pH 7.5, $500 \mathrm{mM} \mathrm{LiCl,} 2 \mathrm{mM} \beta$-mercaptoethanol, $10 \mu \mathrm{M}$ GTP, protease inhibitor, PMSF, and lysosyme. The GST fusion proteins were affinity-purified using gluthatione sepharose 4B (GE Healthcare) and eluted with $50 \mathrm{mM}$ Hepes pH 7.5, $500 \mathrm{mM} \mathrm{LiCl,} 2 \mathrm{mM} \beta$-mercaptoethanol, $10 \mu \mathrm{M}$ $\mathrm{GTP}$, and $15 \mathrm{mM}$ reduced glutathione. Purified proteins were then dialyzed against a $2 \mathrm{~L}$ solution containing $25 \mathrm{mM}$ Hepes pH 7.5, $50 \mathrm{mM} \mathrm{NaCl}, 2 \mathrm{mM} \beta$-mercaptoethanol, $2 \mathrm{mM} \mathrm{MgCl} 2,10 \mu \mathrm{M}$ GTP. 6xHis-wild-type RAB6A was expressed in the BL21 codon plus (DE3)-RIL of E. coli after induction with $400 \mu \mathrm{M}$ isopropyl- $\beta$ d-thiogalactopyranoside at $30^{\circ} \mathrm{C}$ for $4 \mathrm{~h}$. Cells were lysed by cell disruption in $50 \mathrm{mM}$ HEPES pH 7,5, $500 \mathrm{mM} \mathrm{LiCl,} 2 \mathrm{mM} \beta$-mercaptoethanol, $1 \mathrm{mM} \mathrm{MgCl} 2,10$ $\mu \mathrm{M}$ GTP, protease inhibitors, PMSF, and lysosyme. Proteins were affinity-purified using HiTrap ${ }^{\text {n" }}$ Chelating HP (GE Healthcare) and eluted with 50mM Hepes $\mathrm{pH} 7,5,500 \mathrm{mM} \mathrm{LiCl}, 2 \mathrm{mM} \beta$-mercaptoethanol, $1 \mathrm{mM} \mathrm{MgCl} 2,10 \mu \mathrm{M}$ GTP, $500 \mathrm{mM}$ Imidazole. His-tags were removed by recombinant Tobacco Etch Virus (rTEV) protease cleavage during overnight dialysis in $50 \mathrm{mM}$ Hepes $\mathrm{pH}$ 7,5, $100 \mathrm{mM} \mathrm{NaCl}, 2 \mathrm{mM} \beta$-mercaptoethanol, $1 \mathrm{mM} \mathrm{MgCl} 2,10 \mu \mathrm{M}$ GTP. A last step of gel filtration with Superdex 75 16/600 (GE Healthcare) was performed with a buffer containing $25 \mathrm{mM}$ Hepes pH 7.5, $50 \mathrm{mM} \mathrm{NaCl}, 1 \mathrm{mM} \mathrm{MgCl} 2,2 \mathrm{mM}$ DTE, $10 \mu \mathrm{M}$ GTP. 6x-His-KIF20A-529-665 was purified as in ref. ${ }^{49}$. GST-GFP was a kind gift of the recombinant antibody platform (Institut Curie).

Solid-phase assays were carried out as in ref. ${ }^{34}$. Briefly, 96-well plates (Nunc) were coated with $10 \mu \mathrm{g}$ of either GST-GFP, GST-Myosin II-1148-1652, GSTRAB6A:GTP, and 0-40 $\mu$ g recombinant 6xHis-RAB6:GTP or 6xHis-KIF20A-529665 proteins was used. Interactions were performed in a buffer containing $20 \mathrm{mM}$ Hepes, pH 7.5, $100 \mathrm{mM} \mathrm{NaCl}$, and $5 \mathrm{mM} \mathrm{MgCl}$. Protein interaction was revealed by anti- $6 \times$ His antibodies (1:1000), using $100 \mu$ TMD (BD Biosciences) as a chromogenic substrate at room temperature for $10 \mathrm{~min}$. The reactions were stopped by the addition of sulphuric acid. Absorbance was measured at $450 \mathrm{~nm}$ in a plate reader.

Purification, crystallization, and structure determination of RAB6:KIF20A603-665 complex. The KIF20A fragment (603-665) was cloned in pGEXII plasmid, containing a N-terminal GST-tag followed by a rTEV protease cleavage site. RAB6A-Q72L fragment (8-195) was cloned in a modified pACYC plasmid with an introduced rTEV cleavage site after the N-terminal His-tag. The complex was produced by co-expression of GST-KIF20A-603-665 and His-RAB6A-Q72L8-195 in E. coli BL21 (DE3). The complex was purified using Glutathione Sepharose $^{\text {sm }} 4 \mathrm{~B}$ in a batch mode. The complex was eluted by rTEV cleavage and 
subsequently purified by Ni-NTA. Extra RAB6A-Q72L-8-195 was added to the purified complex mix to ensure saturation of KIF20A-RBD with the binding partner.

The complex at concentration of $10 \mathrm{mg} / \mathrm{ml}$ was crystallized in $100 \mathrm{mM}$ Hepes pH 7.4, $200 \mathrm{mM}$ ammonium sulfate, $24 \%$ (w/v) PEG $8 \mathrm{~K}, 2 \%(\mathrm{v} / \mathrm{v})$ isopropanol in hanging drop geometry at $17^{\circ} \mathrm{C}$. A $2 \AA$ resolution data set was collected at the European Synchrotron Radiation Facility (ESRF) synchrotron (beamline ID29). The structure was determined by molecular replacement method using Molrep ${ }^{52}$ and RAB6 structure (PDB ID 2GIL ${ }^{53}$ ) as a search model. The structure was iteratively rebuilt using $\operatorname{Coot}^{54}$ and refined using Phenix ${ }^{55}$ (Supplementary Table 1). The atomic coordinates and structure factors of the structure of the RAB6:KIF20A:RBD complex has been deposited in the Protein Data Bank (www. pdb.org) with accession number PDB ID 5LEF.

Transport assays. For vesicular stomatitis virus G protein (VSV-G)-trafficking experiments, HeLa cells were transfected with a plasmid allowing the expression of the thermosensitive mutant GFP-VSV-G tsO45 and incubated for $14 \mathrm{~h}$ at $40^{\circ} \mathrm{C}$. Transport to the Golgi complex and to the plasma membrane was induced by shifting cells at $32^{\circ} \mathrm{C}$ for indicated times. Arrival of VSV-G at the plasma membrane was monitored by using a mouse monoclonal VG antibody directed against the exoplasmic domain of the protein, in the absence of detergent. Surface VSV-G was then revealed with a Cy3-labeled anti-mouse antibody. Cell to cell variation of the GFP-VSV-G expression levels were taken into account by measuring the ratio between plasma membrane VSV-G (surface labeling described in the Transport assay section) to the total VSV-G (surface + intracellular) after Z-projection of each color channels.

Statistical analysis. All data were generated from cells pooled from at least three independent experiments represented as $(n)$, unless mentioned, in corresponding legends. Statistical data were presented as means \pm standard error of the mean. Statistical significance was determined by Student's $t$ test for two or three sets of data using Excel, no sample was excluded. Cells were randomly selected. Only $P$ value $<0.05$ was considered as statistically significant.

Data availability. The authors declare that all relevant data supporting the findings of this study are available within the paper (and its Supplementary information file). Any raw data can be obtained from the corresponding author (S.M.-L.) on reasonable request.

Received: 7 July 2016 Accepted: 4 September 2017

Published online: 01 November 2017

\section{References}

1. Lowe, M. Structural organization of the Golgi apparatus. Curr. Opin. Cell Biol. 23, 85-93 (2011).

2. Egea, G., Serra-Peinado, C., Salcedo-Sicilia, L. \& Gutiérrez-Martínez, E. Actin acting at the Golgi. Histochem. Cell Biol. 140, 347-360 (2013).

3. Almeida, C. G. et al. Myosin $1 \mathrm{~b}$ promotes the formation of post-Golgi carriers by regulating actin assembly and membrane remodelling at the trans-Golgi network. Nat. Cell Biol. 13, 779-789 (2011).

4. Blume von, J. et al. Actin remodeling by ADF/cofilin is required for cargo sorting at the trans-Golgi network. J. Cell Biol. 187, 1055-1069 (2009).

5. Blume von, J. et al. ADF/cofilin regulates secretory cargo sorting at the TGN via the Ca2+ ATPase SPCA1. Dev. Cell 20, 652-662 (2011).

6. Miserey-Lenkei, S. et al. Rab and actomyosin-dependent fission of transport vesicles at the Golgi complex. Nat. Cell Biol. 12, 645-654 (2010).

7. Müsch, A., Cohen, D. \& Rodriguez-Boulan, E. Myosin II is involved in the production of constitutive transport vesicles from the TGN. J. Cell Biol. 138, 291-306 (1997).

8. Dippold, H. C. et al. GOLPH3 bridges phosphatidylinositol-4- phosphate and actomyosin to stretch and shape the golgi to promote budding. Cell 139, 337-351 (2009).

9. De Matteis, M. A. \& Luini, A. Exiting the Golgi complex. Nat. Rev. Mol. Cell Biol. 9, 273-284 (2008).

10. Zilberman, Y. et al. Involvement of the Rho-mDial pathway in the regulation of Golgi complex architecture and dynamics. Mol. Biol. Cell 22, 2900-2911 (2011).

11. Colón-Franco, J. M., Gomez, T. S. \& Billadeau, D. D. Dynamic remodeling of the actin cytoskeleton by FMNL1 $\gamma$ is required for structural maintenance of the Golgi complex. J. Cell Sci. 124, 3118-3126 (2011).

12. Ramabhadran, V., Korobova, F., Rahme, G. J. \& Higgs, H. N. Splice variantspecific cellular function of the formin INF2 in maintenance of Golgi architecture. Mol. Biol. Cell 22, 4822-4833 (2011).
13. Gardberg, M. et al. Characterization of Diaphanous-related formin FMNL2 in human tissues. BMC Cell Biol. 11, 55 (2010).

14. Campellone, K. G., Webb, N. J., Znameroski, E. A. \& Welch, M. D. WHAMM is an Arp2/3 complex activator that binds microtubules and functions in ER to golgi transport. Cell 134, 148-161 (2008).

15. Papoulas, O., Hays, T. S. \& Sisson, J. C. The golgin Lava lamp mediates dyneinbased Golgi movements during Drosophila cellularization. Nat. Cell Biol. 7, 612-618 (2005).

16. Kakinuma, T., Ichikawa, H. \& Tsukada, Y. Interaction between p230 and MACF1 is associated with transport of a glycosyl phosphatidyl inositolanchored protein from the Golgi to the cell periphery. Exp. Cell 298, 388-398 (2004).

17. Grigoriev, I. et al. Rab6 regulates transport and targeting of exocytotic carriers. Dev. Cell 13, 305-314 (2007).

18. Storrie, B. et al. Electron tomography reveals Rab6 is essential to the trafficking of trans-golgi clathrin and COPI-coated vesicles and the maintenance of golgi cisternal number. Traffic 13, 727-744 (2012).

19. Martinez, O. et al. GTP-bound forms of rab6 induce the redistribution of Golgi proteins into the endoplasmic reticulum. Proc. Natl Acad. Sci. USA 94, 1828-1833 (1997).

20. Martinez, O. et al. The small GTP-binding protein rab6 functions in intra-Golgi transport. J. Cell Biol. 127, 1575-1588 (1994).

21. Mallard, F. Early/recycling endosomes-to-TGN transport involves two SNARE complexes and a Rab6 isoform. J. Cell Biol. 156, 653-664 (2002).

22. Echard, A. et al. Alternative splicing of the human Rab6A gene generates two close but functionally different isoforms. Mol. Biol. Cell 11, 3819-3833 (2000).

23. Lindsay, A. J. et al. Identification and characterization of multiple novel Rabmyosin Va interactions. Mol. Biol. Cell 24, 3420-3434 (2013).

24. Barnekow, A., Thyrock, A. \& Kessler, D. in International Review of Cell and Molecular Biology Vol 274, 235-274 (Elsevier, 2009).

25. Echard, A. et al. Interaction of a Golgi-associated kinesin-like protein with Rab6. Science 279, 580-585 (1998).

26. Short, B., Preisinger, C., Schaletzky, J., Kopajtich, R. \& Barr, F. A. The Rab6 GTPase regulates recruitment of the dynactin complex to Golgi membranes. Curr. Biol. 12, 1792-1795 (2002).

27. Maliga, Z. et al. A genomic toolkit to investigate kinesin and myosin motor function in cells. Nat. Cell Biol. 15, 325-334 (2013).

28. Kitagawa, M., Fung, S. Y. S., Onishi, N., Saya, H. \& Lee, S. H. Targeting Aurora $\mathrm{B}$ to the equatorial cortex by MKlp2 is required for cytokinesis. PLoS ONE $\mathbf{8}$, e64826 (2013).

29. Tcherniuk, S. et al. Relocation of Aurora B and Survivin from centromeres to the central spindle impaired by a kinesin-specific MKLP-2 inhibitor. Angew. Chem. Int. Ed. Engl. 49, 8228-8231 (2010).

30. Logan, K. M. \& Knight, K. L. Mutagenesis of the P-loop motif in the ATP binding site of the RecA protein from Escherichia coli. J. Mol. Biol. 232, 1048-1059 (1993).

31. Li, X. D. et al. Effects of mutations in the gamma-phosphate binding site of myosin on its motor function. J. Biol. Chem. 273, 27404-27411 (1998).

32. Kull, F. J., Vale, R. D. \& Fletterick, R. J. The case for a common ancestor: kinesin and myosin motor proteins and G proteins. J. Muscle Res. Cell Motil. 19, 877-886 (1998).

33. Ikonen, E. et al. Myosin II is associated with Golgi membranes: identification of p200 as nonmuscle myosin II on Golgi-derived vesicle. J. Cell Sci. 110, 2155-2164 (1997).

34. Dambournet, D. et al. Rab35 GTPase and OCRL phosphatase remodel lipids and F-actin for successful cytokinesis. Nat. Cell Biol. 13, 981-988 (2011).

35. Gruneberg, U. et al. Relocation of Aurora B from centromeres to the central spindle at the metaphase to anaphase transition requires MKlp2. J. Cell Biol. 166, 167-172 (2004).

36. Neef, R. et al. Phosphorylation of mitotic kinesin-like protein 2 by polo-like kinase 1 is required for cytokinesis. J. Cell Biol. 162, 863-876 (2003).

37. Neef, R. et al. Choice of Plk1 docking partners during mitosis and cytokinesis is controlled by the activation state of Cdk1. Nat. Cell Biol. 9, 436-444 (2007).

38. Efimov, A. et al. Asymmetric CLASP-dependent nucleation of noncentrosomal microtubules at the trans-Golgi network. Dev. Cell 12, 917-930 (2007).

39. Rivero, S., Cardenas, J., Bornens, M. \& Rios, R. M. Microtubule nucleation at the cis-side of the Golgi apparatus requires AKAP450 and GM130. EMBO J. 28, 1016-1028 (2009).

40. Miller, P. M. et al. Golgi-derived CLASP-dependent microtubules control Golgi organization and polarized trafficking in motile cells. Nat. Cell Biol. 11, 1069-1080 (2009).

41. Keller, P., Toomre, D., Díaz, E., White, J. \& Simons, K. Multicolour imaging of post-Golgi sorting and trafficking in live cells. Nat. Cell Biol. 3, 140-149 (2001).

42. Pfeffer, S. R. Rab GTPase localization and Rab cascades in Golgi transport. Biochem. Soc. Trans. 40, 1373-1377 (2012).

43. Bonazzi, M. et al. CtBP3/BARS drives membrane fission in dynaminindependent transport pathways. Nat. Cell Biol. 7, 570-580 (2005). 
44. Valente, C. et al. A 14-3-3 $\gamma$ dimer-based scaffold bridges CtBP1-S/BARS to PI (4)KIII $\beta$ to regulate post-Golgi carrier formation. Nat. Cell Biol. 14, 343-354 (2012).

45. Pagliuso, A. et al. Golgi membrane fission requires the CtBP1-S/BARS-induced activation of lysophosphatidic acid acyltransferase $\delta$. Nat. Commun. 7, 12148 (2016).

46. Bardin, S. et al. Phenotypic characterisation of RAB6A knockout mouse embryonic fibroblasts. Biol. Cell 107, 427-439 (2015).

47. Goud, B., Zahraoui, A., Tavitian, A. \& Saraste, J. Small GTP-binding protein associated with Golgi cisternae. Nature 345, 553-556 (1990).

48. Del Nery, E. et al. Rab6A and Rab6A' GTPases play non-overlapping roles in membrane trafficking. Traffic 7, 394-407 (2006).

49. Echard, A., Marjou, El, A. \& Goud, B. Expression, purification, and biochemical properties of rabkinesin- 6 domains and their interactions with Rab6A. Meth. Enzymol 329, 157-165 (2001).

50. Nizak, C. et al. Recombinant antibodies against subcellular fractions used to track endogenous Golgi protein dynamics in vivo. Traffic. 4, 739-753 (2003).

51. Loiodice, I. et al. The entire Nup107-160 complex, including three new members, is targeted as one entity to kinetochores in mitosis. Mol. Biol. Cell 15, 3333-3344 (2004).

52. Vagin, A. \& Teplyakov, A. Molecular replacement with MOLREP. Acta Crystallogr. D Biol. Crystallogr. 66, 22-25 (2010).

53. Bergbrede, T. et al. Biophysical analysis of the interaction of Rab6a GTPase with its effector domains. J. Biol. Chem. 284, 2628-2635 (2009).

54. Emsley, P. \& Cowtan, K. Coot: model-building tools for molecular graphics. Acta Crystallogr. D Biol. Crystallogr. 60, 2126-2132 (2004).

55. Adams, P. D. et al. PHENIX: a comprehensive Python-based system for macromolecular structure solution. Acta Crystallogr. D Biol. Crystallogr. 66, 213-221 (2010).

56. Hill, E., Clarke, M. \& Barr, F. A. The Rab6-binding kinesin, Rab6-KIFL, is required for cytokinesis. EMBO J. 19, 5711-5719 (2000).

57. Fontijn, R. D. et al. The human kinesin-like protein RB6K is under tight cell cycle control and is essential for cytokinesis. Mol. Cell Biol. 21, 2944-2955 (2001)

\section{Acknowledgements}

We are grateful to Franck Perez and Ana-Maria Lennon-Duménil (Institut Curie) for generous gifts of reagents; Ana-Maria Lennon-Duménil, Yohanns Bellaïche, and Franck Perez for critical reading of the manuscript and insightful discussions; Carina Santos, Laura Picas and Jean-Baptiste Brault and the Molecular Mechanisms Transport lab for productive discussions. The authors greatly acknowledge the Nikon Imaging Center at Institut Curie-CNRS, the PICT-IBiSA, member of the France-BioImaging national research infrastructure. This work was supported by an ERC (European Research Council) advanced grant (project 339847 "MYODYN") and an ARC grant (SL220120605302). We acknowledge the recombinant protein and antibody platform of the Institut Curie (http://umr144.curie.fr/en/plateform/protein-and-antibody-laboratory001279) for the production of human recombinant antibodies against Giantin, MHC, and tubulin. The Goud and Houdusse teams are member of Labex CelTisPhyBio (11-LBX-0038) and Idex Paris Sciences et Lettres (ANR-10-IDEX-0001-02 PSL). We thank the beamline scientists of ID29 (ESRF synchrotron) for excellent support during data collection. O.P. was supported by an ARC post-doctoral fellowship. A.H. was supported by grants from the CNRS, ANR-10-MotoRab, ARC PJA 20151203285, ANR15-CE13-0016-01, Ligue Nationale Contre le Cancer.

\section{Author contributions}

S.M.L. carried out the experiments presented in Figs. 1, 2a,d,e, 3, 4d-g, 5, 6b, Supplementary Figs. 1-7 with the help of S.B.; H.B. the experiments in Fig. $4 \mathrm{~h}$ and constructs in Supplementary Figs. 3, 4; A.D. the experiments in Figs. 2c, 6a; V.F. helped for microscopes development and image analysis; G.B. and O.P. determined the X-ray structure with the help of R.B.; O.P., G.B. and A.H. analysed the structure; C.B. and C.G. designed, synthesized and developed paprotrain and BKS0349; S.M.L., A.E., A.H., B.G., O.P. conceived the project; S.M.L., H.B., A.D., A.E., A.H., B.G., O.P. designed and interpreted the experiments; S.M.L., O.P., A.E., A.H., B.G. wrote the manuscript. S.M.L., H.B., O.P., S.B., A.D., V.F., C.G., C.B., A.H., A.E. and B.G. edited the manuscript. B.G and A.H. secured funding.

\section{Additional information}

Supplementary Information accompanies this paper at doi:10.1038/s41467-017-01266-0.

Competing interests: The authors declare no competing financial interests.

Reprints and permission information is available online at http://npg.nature.com/ reprintsandpermissions/

Publisher's note: Springer Nature remains neutral with regard to jurisdictional claims in published maps and institutional affiliations.

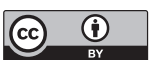

Open Access This article is licensed under a Creative Commons Attribution 4.0 International License, which permits use, sharing, adaptation, distribution and reproduction in any medium or format, as long as you give appropriate credit to the original author(s) and the source, provide a link to the Creative Commons license, and indicate if changes were made. The images or other third party material in this article are included in the article's Creative Commons license, unles indicated otherwise in a credit line to the material. If material is not included in the article's Creative Commons license and your intended use is not permitted by statutory regulation or exceeds the permitted use, you will need to obtain permission directly from the copyright holder. To view a copy of this license, visit http://creativecommons.org/ licenses/by/4.0/.

(C) The Author(s) 2017 